\title{
An AGN sample with high X-ray-to-optical flux ratio from RASS
}

\section{The optical identification}

\author{
J.Y. Wei ${ }^{1,2}$, D.W. Xu ${ }^{1,2}$, X.Y. Dong ${ }^{1,2}$, and J.Y. Hu ${ }^{1,2}$ \\ 1 Beijing Astronomical Observatory, Chinese Academy of Sciences, Beijing 100012, PR China \\ 2 National Astronomical Observatories, Chinese Academy of Sciences, Beijing 100012, PR China
}

Received June 21; accepted August 26, 1999

\begin{abstract}
We present the first results of a program to identify the unknown bright active galactic nuclei (AGN) in the ROSAT All Sky Survey Bright Source Catalog (RASS-BSC). We have used $\log C+0.4 R$ as an alternative expression for $\log \left(f_{\mathrm{X}} / f_{\text {opt }}\right)$, where $C$ is X-ray count rate and $R$ stands for $R$ magnitude. Then a high $\mathrm{X}$-rayto-optical flux ratio criterion has been used to select an AGN sample with 165 unidentified X-ray sources for optical spectroscopy. Those $165 \mathrm{X}$-ray sources have been identified in the following classes: 115 emission line AGN (QSOs and Seyferts), 2 BL Lac objects and 4 BL Lac candidates, 22 clusters of galaxies, 12 Galactic stars and 10 objects remain unidentified. This represent a success rate of about $73 \%$ for detecting AGN using our selection criteria. Plausibility is based upon the optical classification and X-ray characteristics of the sources.
\end{abstract}

Key words: surveys - X-rays: general - X-rays: galaxies - galaxies: active

\section{Introduction}

During the ROSAT All-Sky Survey (RASS; Voges 1992, 1997) about 80000 X-ray sources with a detection likelihood $\geq 10$ were detected, from which 18811 sources having a PSPC count rate larger than $0.05 \mathrm{cts}^{-1}$ and detection likelihood $\geq 15$ were compiled in the RASS-BSC (Voges et al. 1996a). More than 65\% RASS sources remain unidentified (Voges et al. 1996b). The optical identification of this large numbers of X-ray sources is a challenge. In order to obtain the statistical classification of the RASS sources, several optical identification programs have been carried out (Appenzeller et al. 1998; Fischer et al. 1998; Hasinger et al. 1998; Schmidt et al. 1998; Thomas et al. 1998).
The identification of X-ray sources has proved to be an effective means of creating samples of Active galactic nuclei (AGN). The present program aims at creating a new bright sample of AGN which have the highest X-ray-tooptical flux ratios for statistical investigation. The results of the EMSS (Stocke et al. 1991) have shown that different classes of X-ray sources represent different narrow ranges in the X-ray-to-optical flux ratios. These bounds mean the X-ray sources with Galactic and extragalactic counterparts can be separated at high confidence level prior to any optical spectroscopy (Maccacaro et al. 1988).

Sections 2 and 3 review the creation and follow-up optical spectroscopic observations of the sample. Section 4 gives details of the optical identification procedure. The final section presents the results. A Hubble constant of $H_{0}=50 \mathrm{~km} \mathrm{~s}^{-1} \mathrm{Mpc}^{-1}$ and cosmological deceleration parameter of $q_{0}=0$ are used throughout.

\section{Sample selection}

The alternative expression for X-ray-to-optical flux ratio $\log \left(f_{X} / f_{\text {opt }}\right)$ comes from the statistical analysis of known RASS-BSC X-ray sources. The sources which have an entry in SIMBAD, NED, the catalogs of AGN (Véron-Cetty \& Véron 1996), or other available databases are considered as known.

The X-ray sources with the highest X-rayto-optical flux ratio are: BL Lac objects, emission line AGN, clusters of galaxies etc. However, $\mathrm{X}$-ray flux is difficult to evaluate before optical identification. Although for a given spectral energy distribution the conversion of $\mathrm{X}$-ray count rate to flux depends on the column density $N_{\mathrm{H}}$, the X-ray flux $\left(f_{\mathrm{X}}\right)$ is nevertheless roughly proportional to the count rate $(C)$, so X-ray flux in the X-ray-to-optical flux ratio criterion can be replaced by X-ray count rate, i.e., $\log \left(f_{\mathrm{X}} / f_{\text {opt }}\right) \propto \log C+0.4 R+$ constant, where we use the $R$ magnitude flux to represent the optical flux. For fixed $\log \left(f_{\mathrm{X}} / f_{\text {opt }}\right), \log C+0.4 R=$ 


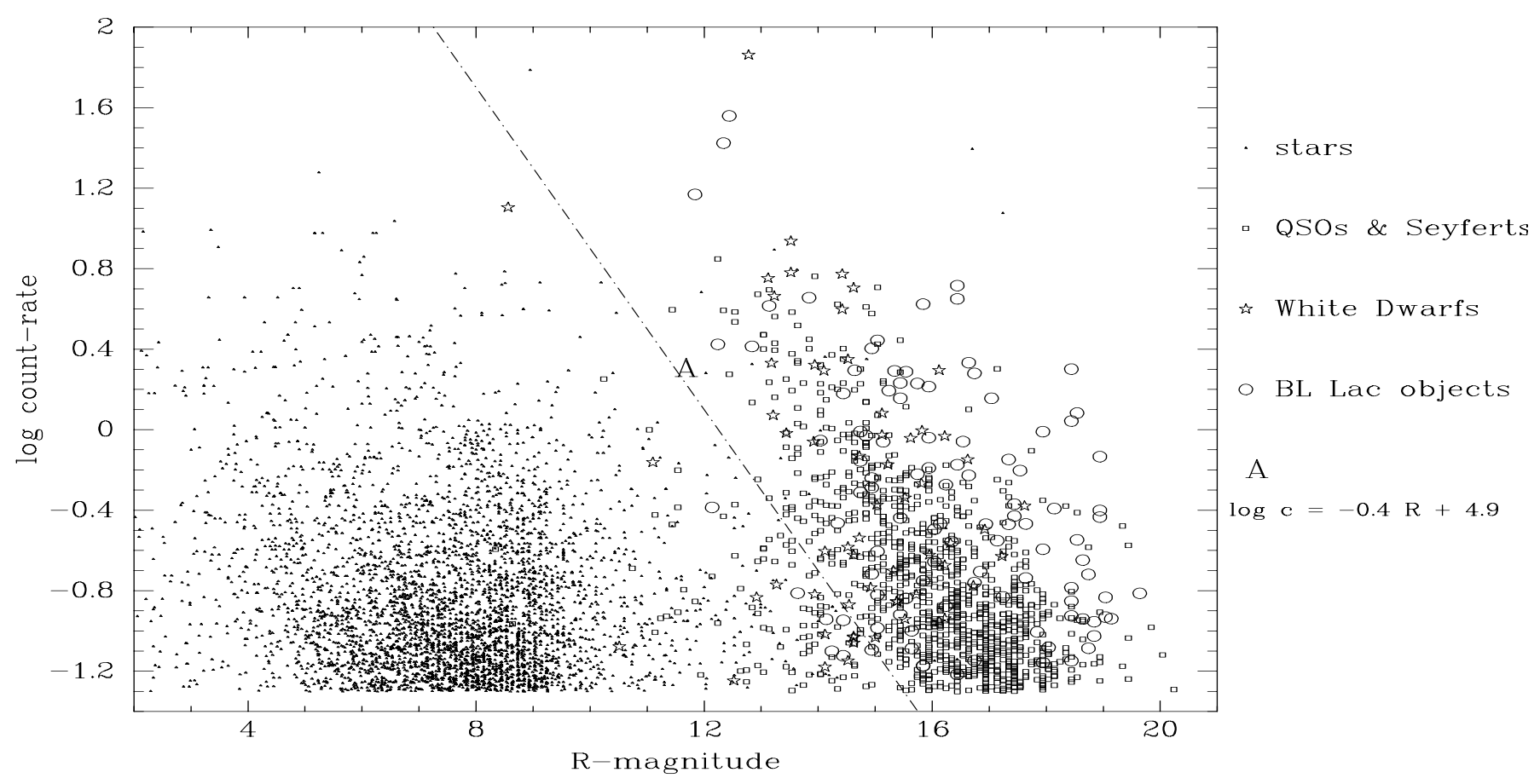

Fig. 1. Count rates as a function of $R$ magnitudes for known sources

constant. According to our statistical analysis of known RASS-BSC X-ray sources, we found that there is an apparent gap between Galactic stars and extragalactic objects. As can be seen in Fig. 1, 92\% AGN concentrate in the region: $\log C \geq-0.4 R+4.9$. O-M spectral type stars in this region are only $3 \%$ of its total amount included in the statistics. Thus, choosing $\log C \geq-0.4 R+4.9$ is expected to be efficient for the preselecting of AGN and can avoid too many $\mathrm{O}-\mathrm{M}$ stars being included in the sample.

Considering the observatory site, instruments and possible observation times, we selected our sample from RASS-BSC according to the following criteria:

1. Unknown sources which do not have an entry in SIMBAD, NED, or other available databases.

2. Declination $\delta \geq 3^{\circ}$.

3. Galactic latitude $|b| \geq 20^{\circ}$.

4. Optical counterparts within a circle with radius $r=$ $r_{1}+5^{\prime \prime}$, where $r_{1}$ is the RASS position error given by Voges et al. (1996).

5. Optical counterparts with $R$ magnitudes between 13.5 and 16.5 .

6. $\log C \geq-0.4 R+4.9$.

For the search of optical counterparts we adopt the error circle with radius $r, 87 \%$ of known AGN are found within $r$ from X-ray position. For each object inside the error circle, we obtain its $\mathrm{R}$ magnitude from USNO-A1.0 ${ }^{1}$ and check whether it satisfies the flux ratio criterion. Only $\mathrm{X}$-ray sources which have at least one object inside the

1 USNO-A1.0, 1996, CD-ROM version, U.S. Naval Observatory. error circle which meets the criterion are included in the sample. A sample with 165 unidentified X-ray sources with Right Ascension between $19^{\mathrm{h}}$ and $8^{\mathrm{h}}$ was selected for optical spectroscopy from RASS-BSC. Table 2 summarizes the general information for the observed objects as following:

Column (1): ROSAT (1RXS J) source designation.

Columns (2) \& (3): Optical right ascension $\alpha$ and declination $\delta$ of the proposed optical counterpart ${ }^{2}$ (epoch 2000.0) derived from the Digitized Sky Survey (DSS) ${ }^{3}$ images which should be better than $2^{\prime \prime}$ (Irwin et al. 1994). Column (4): ROSAT X-ray position error as given in RASS-BSC.

Column (5): Angular Separation between the X-ray source and the proposed optical counterpart in arcsecond. Column (6): ROSAT total count rate (in counts $\mathrm{s}^{-1}$ ) as given in RASS-BSC.

Column (7): Hardness ratio $H R 1=(B-A) /(B+A)$, where $A$ means the count rate in the energy range $0.1-0.4 \mathrm{keV}$ and $B$ in the energy range $0.5-2.0 \mathrm{keV}$.

Column (8): Hardness ratio $H R 2=(D-C) /(D+C)$, where $C$ means the count rate in the energy range $0.5-0.9 \mathrm{keV}$ and $D$ in the energy range $0.9-2.0 \mathrm{keV}$.

Column (9): Extent in arcsecond as given in RASS-BSC. Columns (10) $\&$ (11): The $R$ and $B$ magnitudes obtained from USNO-A1.0 with an accuracy between 0.25 and 0.40

\footnotetext{
${ }^{2}$ In cases which final identification is not the proposed one, we list information for the real counterpart too.

3 The Digitized Sky Survey, 1994, CD-ROM Version, Space Telescope Science Institute.
} 
magnitudes depending on the declination (Monet et al. 1996).

\section{Optical spectroscopic observations and data reduction}

All sources were investigated during several observing runs performed at Xinglong station, Beijing Astronomical Observatory (BAO) from November 1996 through September 1998. The low resolution spectra were taken with the BAO $2.16 \mathrm{~m}$ telescope and the OMR spectrograph, using a Tektronix $1024 \times 1024 \mathrm{CCD}$ as detector. Two gratings of $300 \mathrm{~g} \mathrm{~mm}^{-1}$ and $150 \mathrm{~g} \mathrm{~mm}^{-1}$ were employed in order to get large wavelength coverage. All observations were made through a $2.3^{\prime \prime}$ slit which produced a resolution of 10 to $11 \AA$ and 20 to $22 \AA$ as measured from the comparison spectra. Exposure time was generally between $1200 \mathrm{~s}$ and $3600 \mathrm{~s}$ depending on the brightness of the object. For a few objects, observations were performed twice in order to get a higher $\mathrm{S} / \mathrm{N}$ ratio spectrum. All observations were obtained at airmass less than 1.5 .

The raw two-dimensional data were reduced following standard procedures using the IRAF program package. The CCD reductions included bias subtraction, flatfield correction and cosmic-ray removal. Wavelength calibration was carried out using helium-neon-argon lamps taken at the beginning and end of the night. The flux calibration was derived with 2 to 3 observations of KPNO standard stars (Massey \& Strobel 1988) per night. The atmospheric extinction was corrected by using the mean extinction coefficients for Xinglong Station, which were measured by Beijing- Arizona-Taiwan-Connecticut (BATC) multi-color survey (Yan 1995). The telluric $\mathrm{O}_{2}$ absorption bands at $6870 \AA$ and $7620 \AA$ were not removed. The final calibrated spectra are shown in Fig. 2. Some of the spectra were taken under nonphotometric conditions so that the flux density scale given in the figures should be used only as a rough guide to the brightness of the sources.

\section{Criteria for classification}

The optical spectra are classified in a manner similar to related optical identification programs of X-ray sources (Stocke et al. 1991; Appenzeller et al. 1998). In the present investigation the plausibility is based on the optical classification and X-ray characteristics like hardness ratios and extension parameter. In many cases an unambiguous identification of an object as optical counterpart is possible using only the spectroscopy and imaging information from the POSS. However, we found a few cases where the optical classification alone does not allow a decision whether the proposed optical counterpart to the X-ray source is a plausible one. In these special cases additional information on the nature of the X-ray sources derived from RASSBSC was used to check the plausibility. Bade et al. (1994) discussed the X-ray properties of white dwarfs (very soft $\mathrm{X}$-ray colors) and nearby clusters of galaxies (hard and extended X-ray emission). Pietsch et al. (1998) also used a conservative extent criterion (extent likelihood $>10$ and extent $>30^{\prime \prime}$ ) as an indicator that the X-ray emission does not originate from a nuclear source.

The redshifts of the spectral features are used to discriminate between X-ray sources with Galactic and extragalactic counterparts. Since our spectral resolution is insufficient to detect emission components in the Ca II resonance lines, we use Balmer emission components as tracers for coronal activity. Among 12 Galactic stars, 3 are M stars with molecular absorption bands, 3 are white dwarfs with very blue continuum and broad Balmer lines in absorption, and 6 are cataclysmic variable stars with Balmer emission lines present in the spectra.

Extragalactic counterparts in the sample are classified with emission line AGN, clusters of galaxies, BL Lac objects and their candidates. Generally, normal galaxies are not expected to be strong X-ray emitters and plausible candidates for the sample's counterparts. Objects with broad $\left(F W H M>1000 \mathrm{~km} \mathrm{~s}^{-1}\right)$ and strong $\left(W_{\lambda} \gg 5 \AA\right)$ permitted emission lines are classified as emission line AGN. The spectroscopic and imaging criteria required to identify an X-ray source as a cluster of galaxies involve the spectrum of a normal galaxy and visual evidence for the cluster on the POSS.

Indications pointing to a BL Lac nature of a specific source are: absence of emission lines with $W_{\lambda} \geq$ $5 \AA$, contrast of the CaII break from the host galaxy small than $25 \%$. Four objects with seemingly normal galaxy spectra are classified as BL Lac candidates due to their centimeter radio fluxes, high X-ray luminosities and consistent $\left(\alpha_{\mathrm{ox}}, \alpha_{\mathrm{ro}}\right)$ values. The use of the $\alpha_{\mathrm{ox}}-$ $\alpha_{\text {ro }}$ diagram for classification was discussed by Stocke et al. (1991) and Nass et al. (1996). It was noticed that X-ray selected BL Lac objects contain a greater percentage of starlight due to the underlying galaxy than radio-selected BL Lac objects. Browne \& Marchã (1993) also pointed out the possibility of misclassified X-ray sources as elliptical galaxies or clusters of galaxies. We tried without success to find other plausible counterparts since other objects visible on the POSS within the error circle are too faint to be observed with the $2.16 \mathrm{~m}$ telescope. However, we can not exclude that the counterparts are among these faint objects. Further observations, imaging and spectroscopy, are needed in these fields. Table 1 gives the X-ray parameters for these four BL Lac candidates. The X-ray flux is the unabsorbed flux in units of $10^{-12} \mathrm{ergs} \mathrm{cm}^{-2} \mathrm{~s}^{-1}$ and has been computed assuming a power law spectrum and Galactic absorption. The radio fluxes at $1.4 \mathrm{GHz}$ are taken from the NRAO VLA Sky Survey (NVSS, Condon et al. 1998). The twopoint spectral indices $\alpha_{\mathrm{ox}}=-\log \left(S_{2 \mathrm{keV}} / S_{2500} \AA\right) / 2.605$ and $\alpha_{\mathrm{ro}}=\log \left(S_{5 \mathrm{GHz}} / S_{2500 \AA} \AA\right) / 5.38$ (Stocke et al. 1985; Tananbaum et al. 1979) have been K-corrected for the 

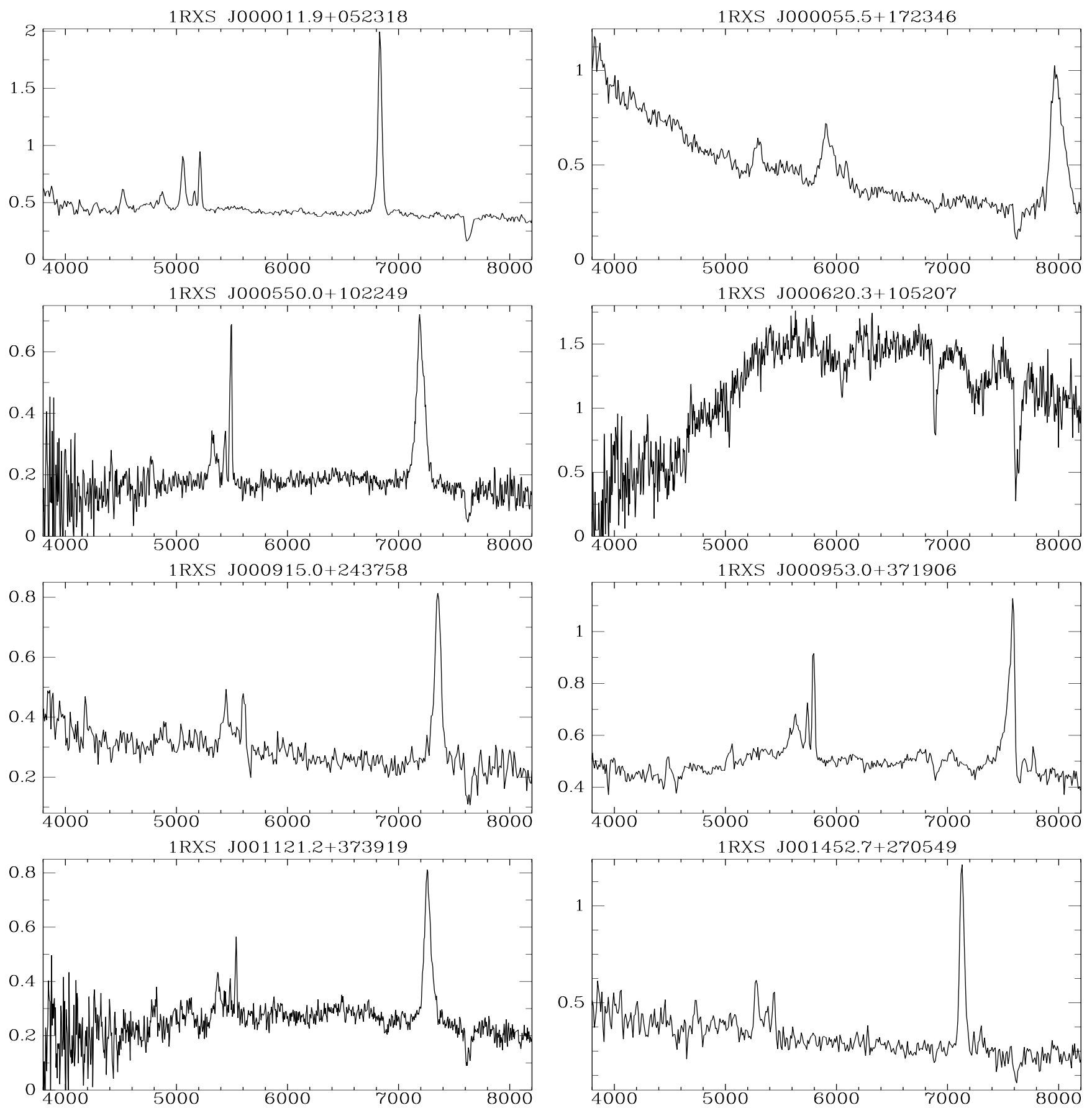

1 RXS J001452.7+270549

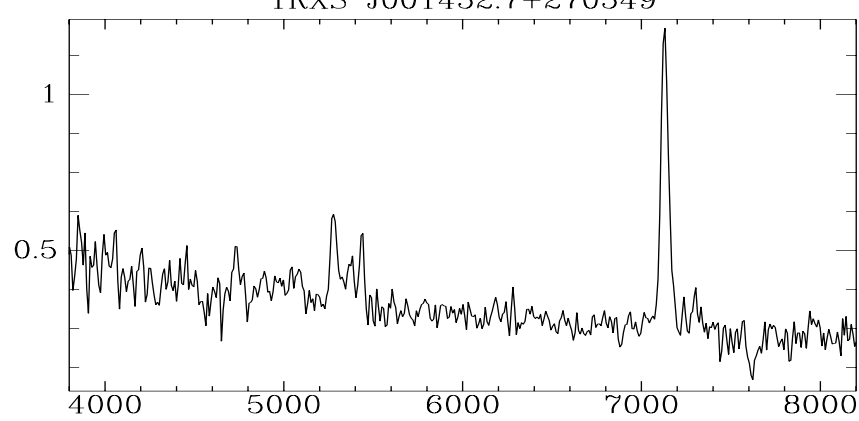

1 RXS J002007.2+324423
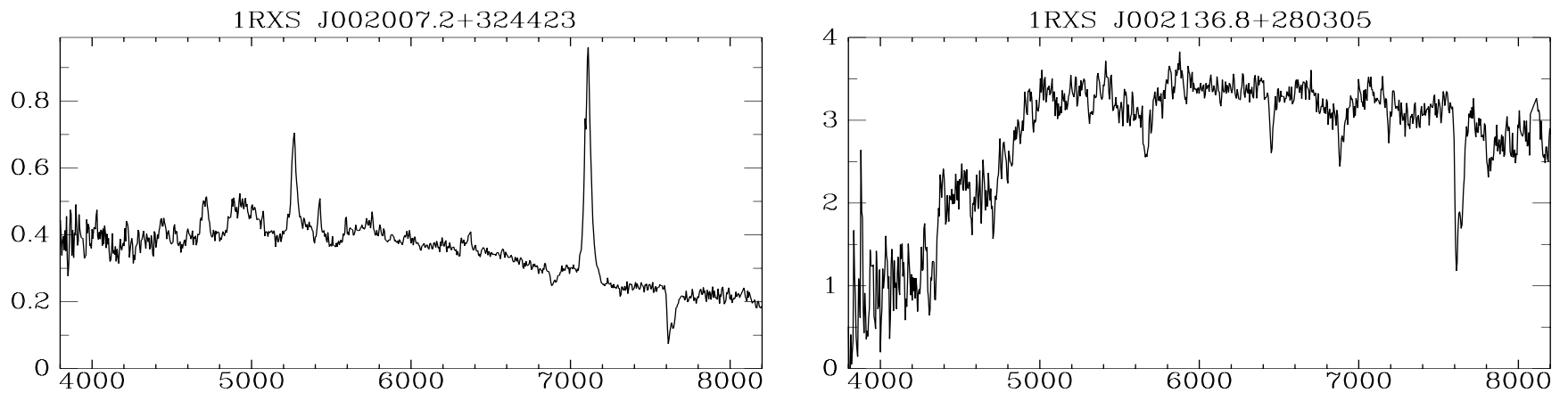

Fig. 2. Optical spectra of the counterparts to the X-ray sources. $f_{\lambda}$ in units of $10^{-15} \mathrm{erg} \mathrm{cm}^{-2} \mathrm{~s}^{-1} \AA^{-1}$ is plotted against wavelength in $\AA$ 

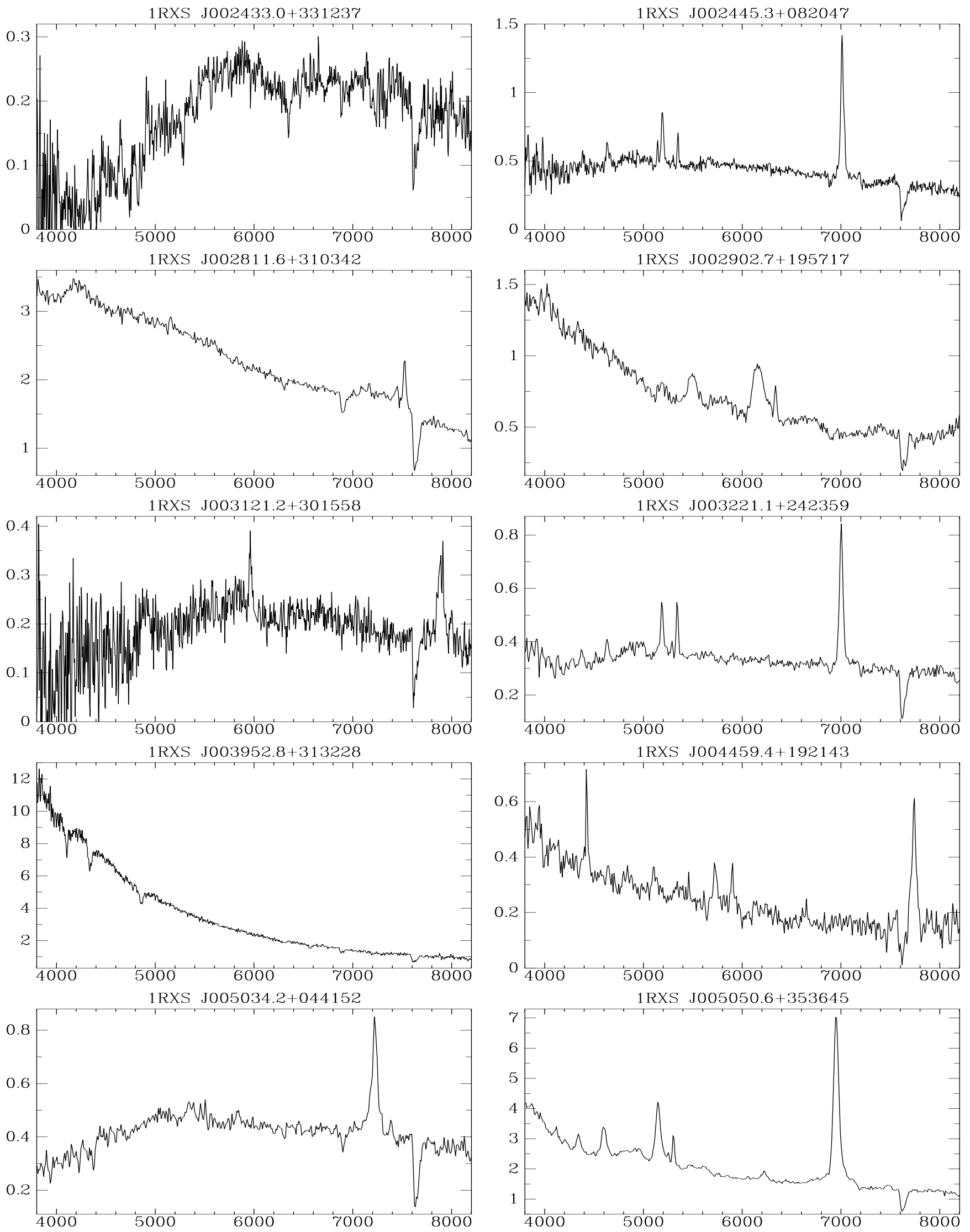

Fig. 2. continued 

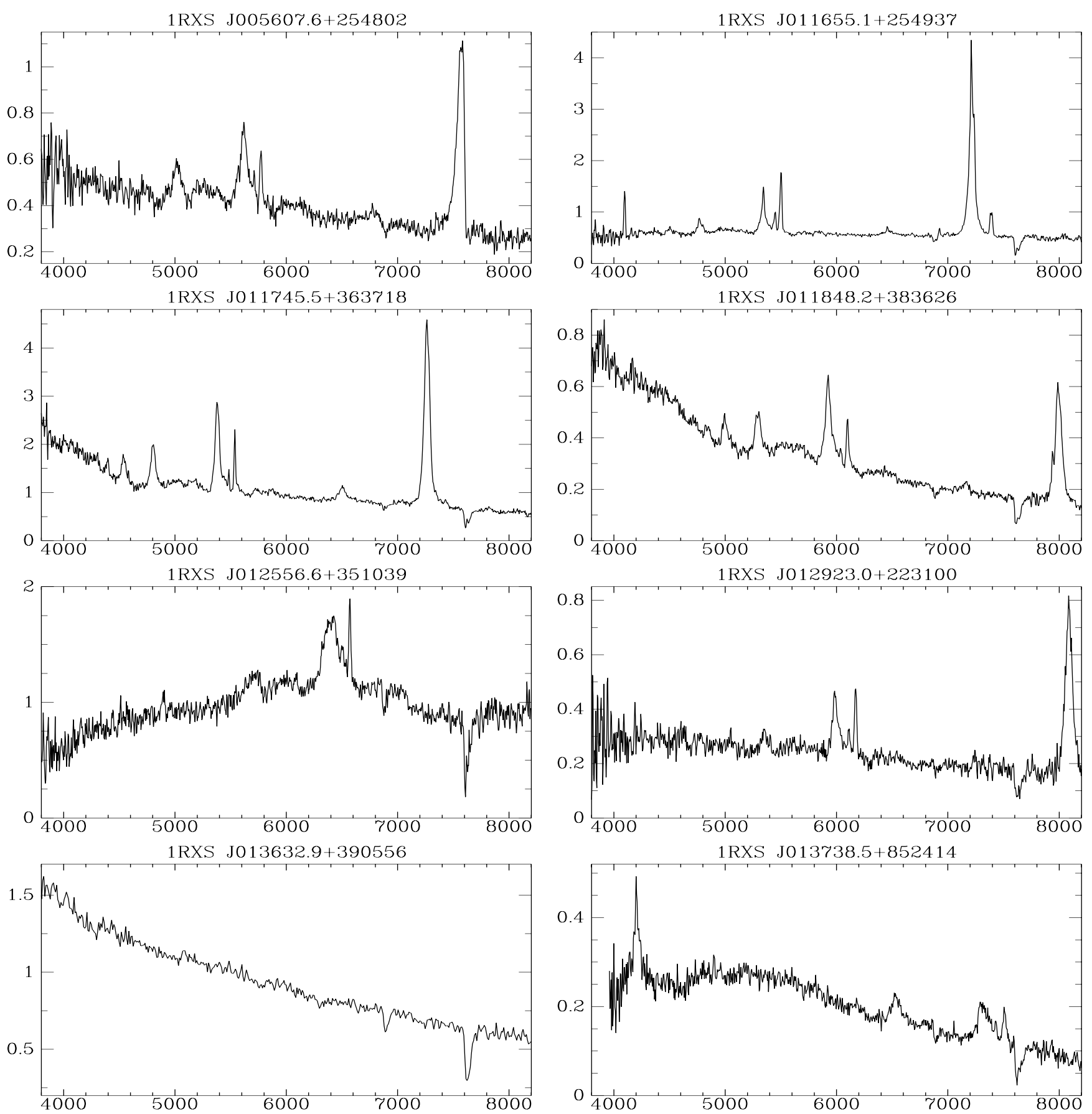
1RXS J013853.1+252325
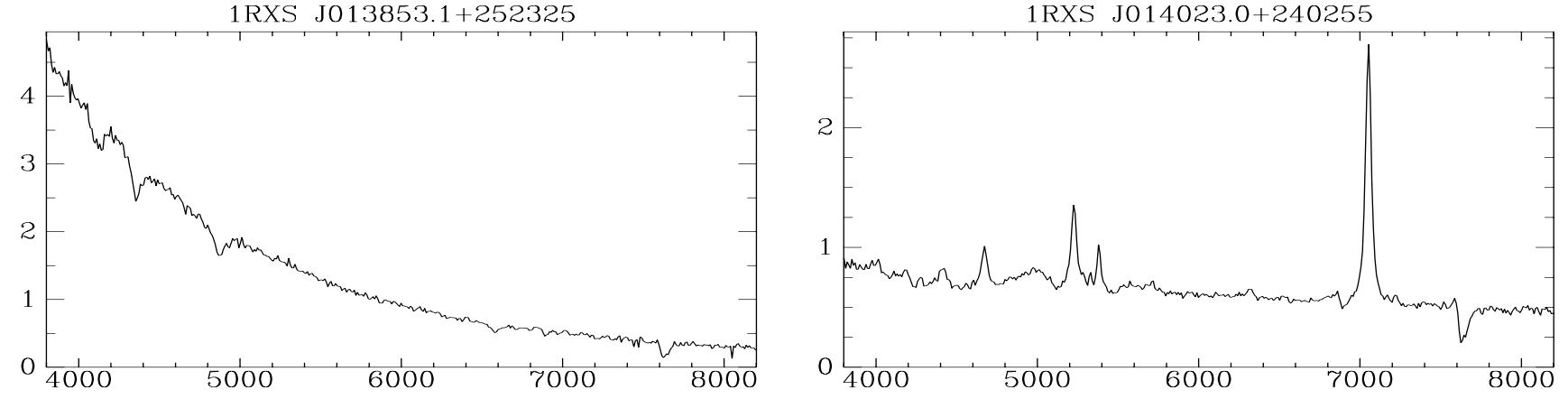

Fig. 2. continued 
1RXS J014025.2+382400

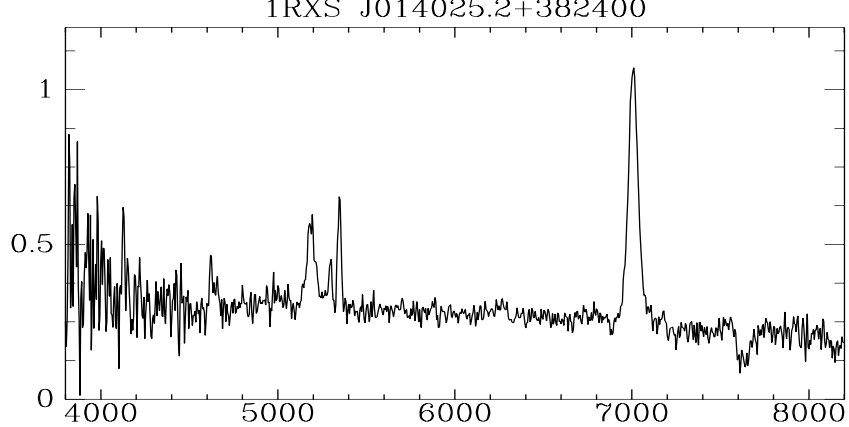

1 RXS J015505.4+335452
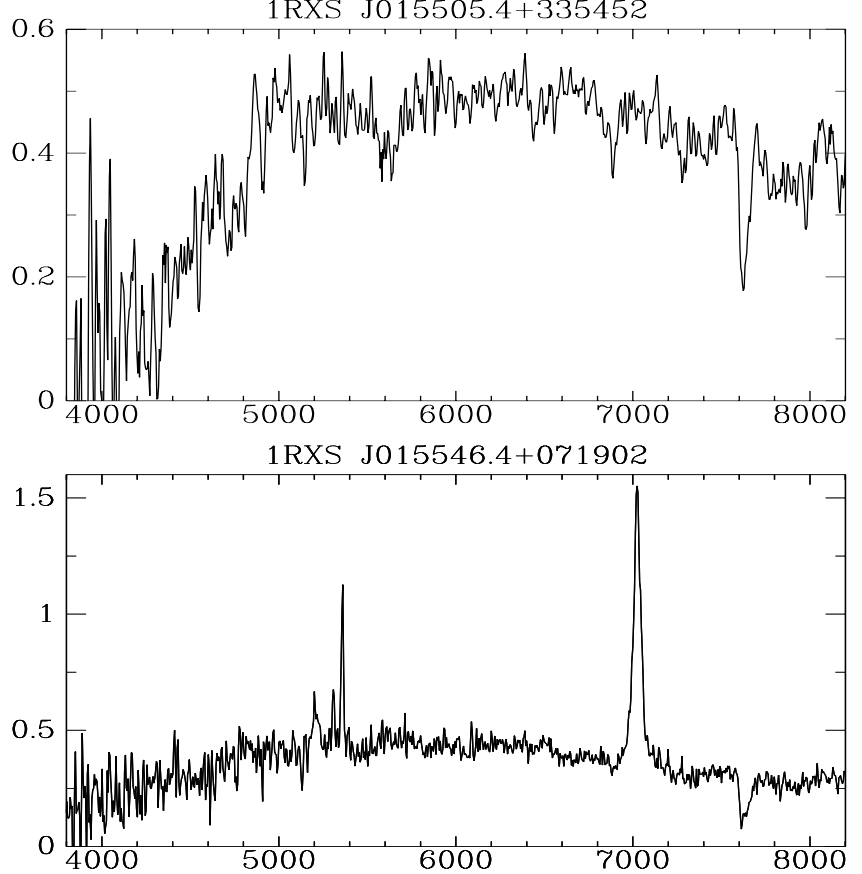

1 RXS J015621.6+241838

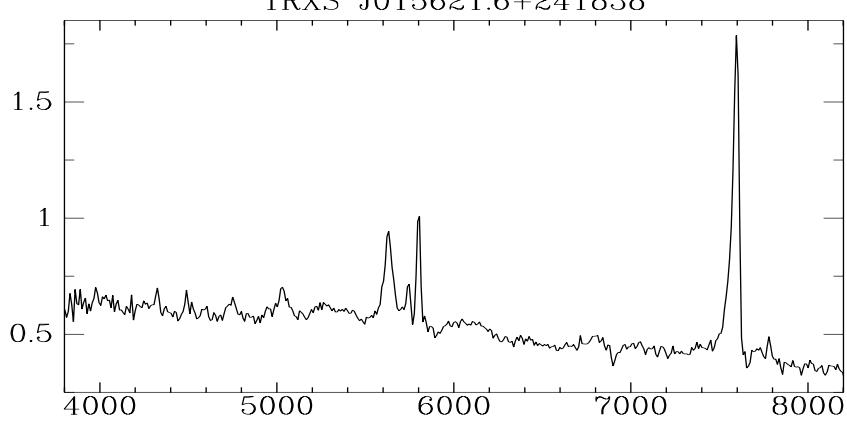

1 RXS Jo22851.6+335949

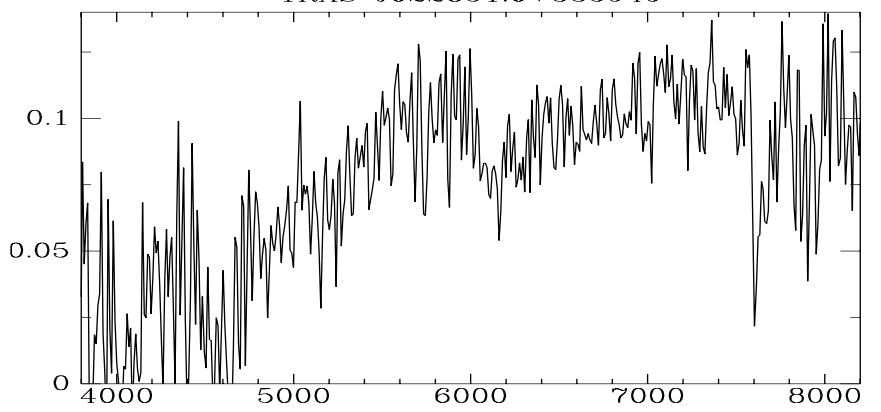

1 RXS J014732.3+345358

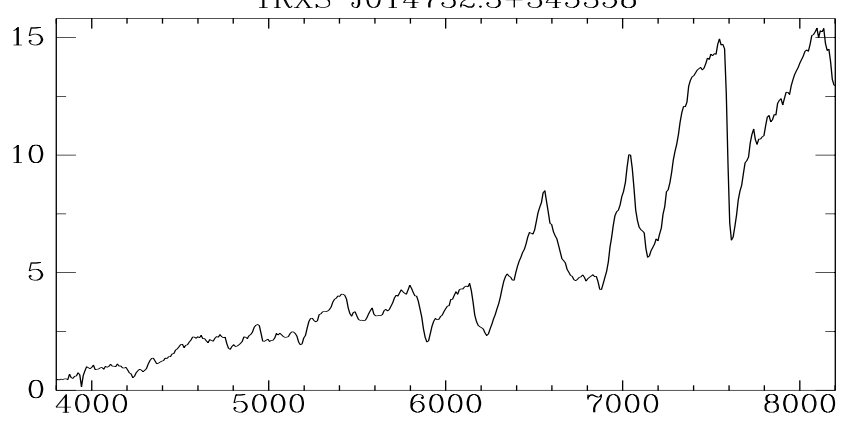

1 RXS J015536.7+311525

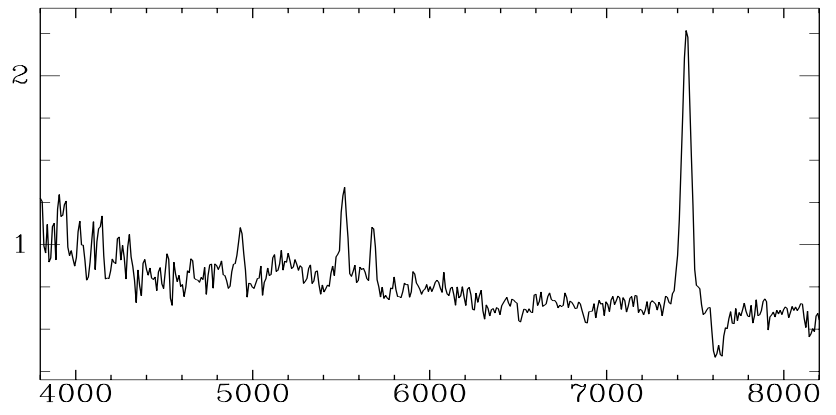

1 RXS J015555.6+040620

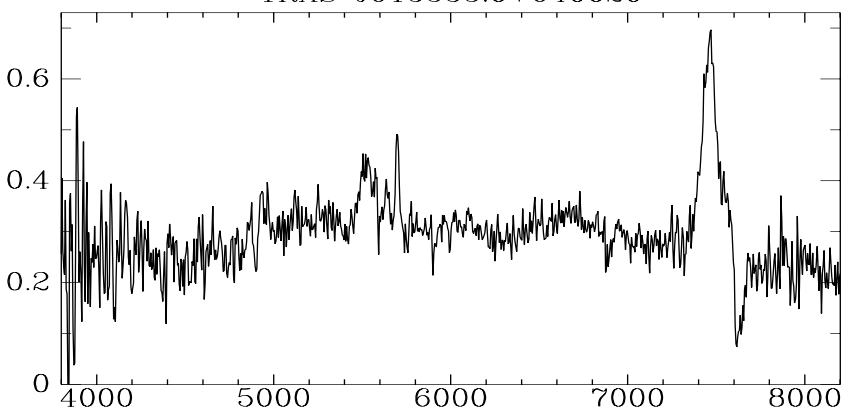

1 RXS J020348.7+295921

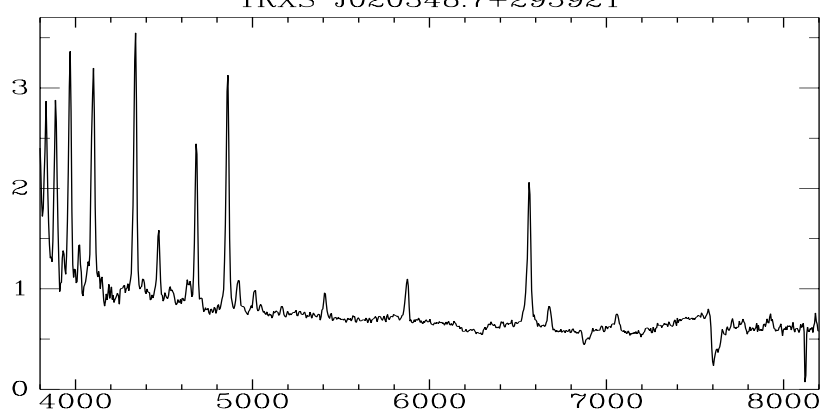

1 RXS J023601.9+082124

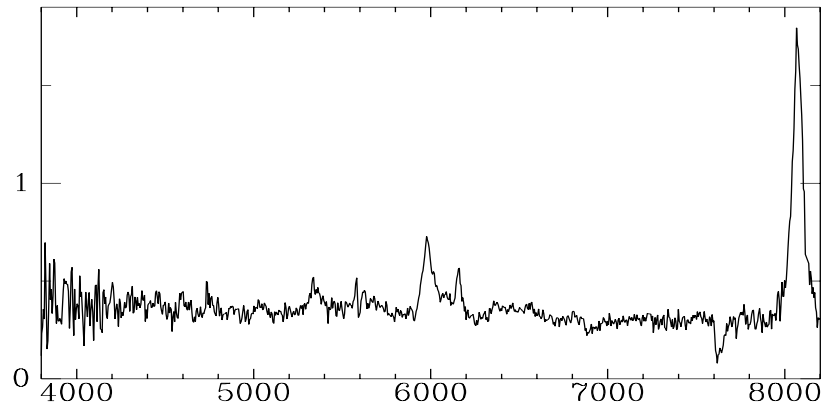

Fig. 2. continued 

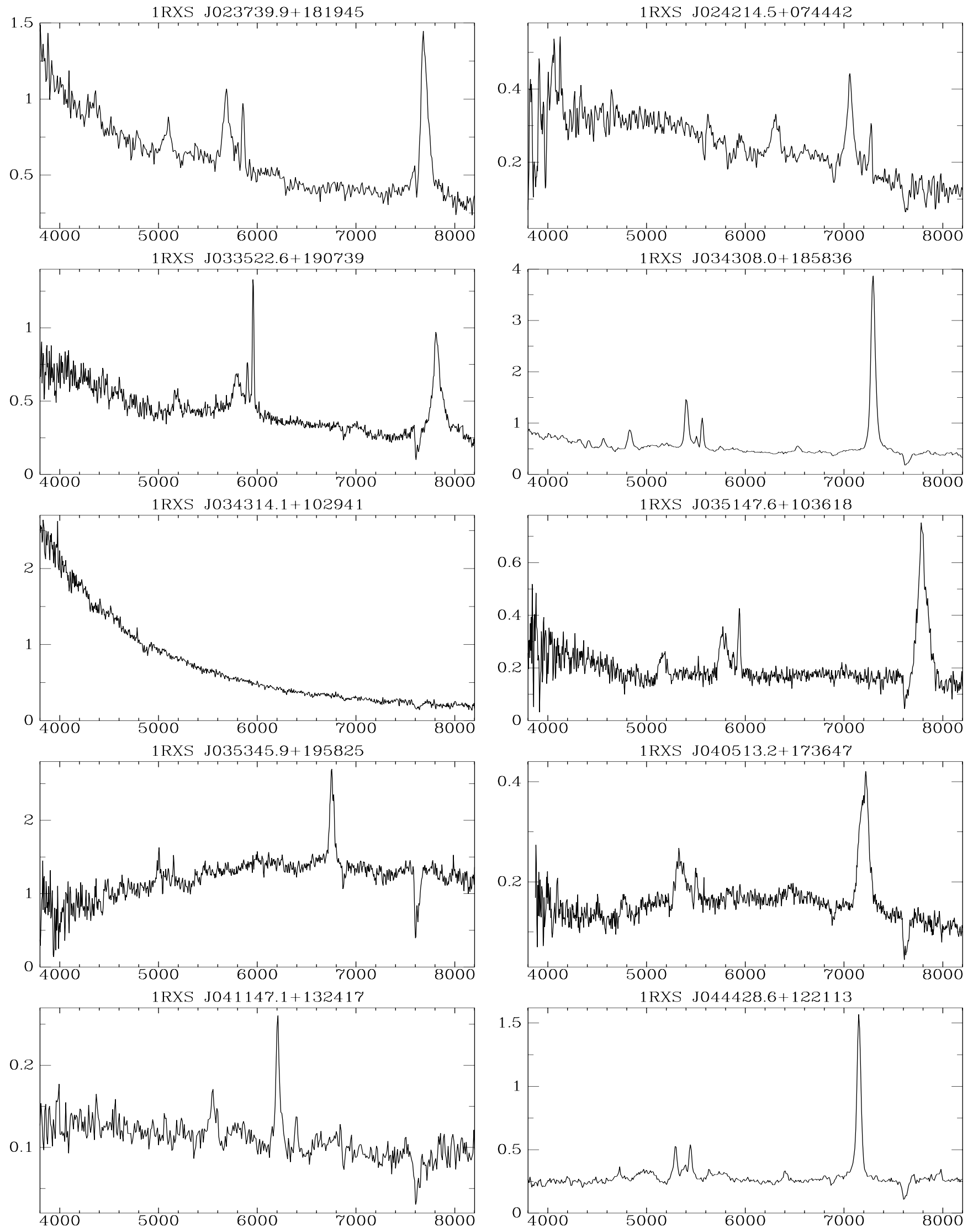

Fig. 2. continued 

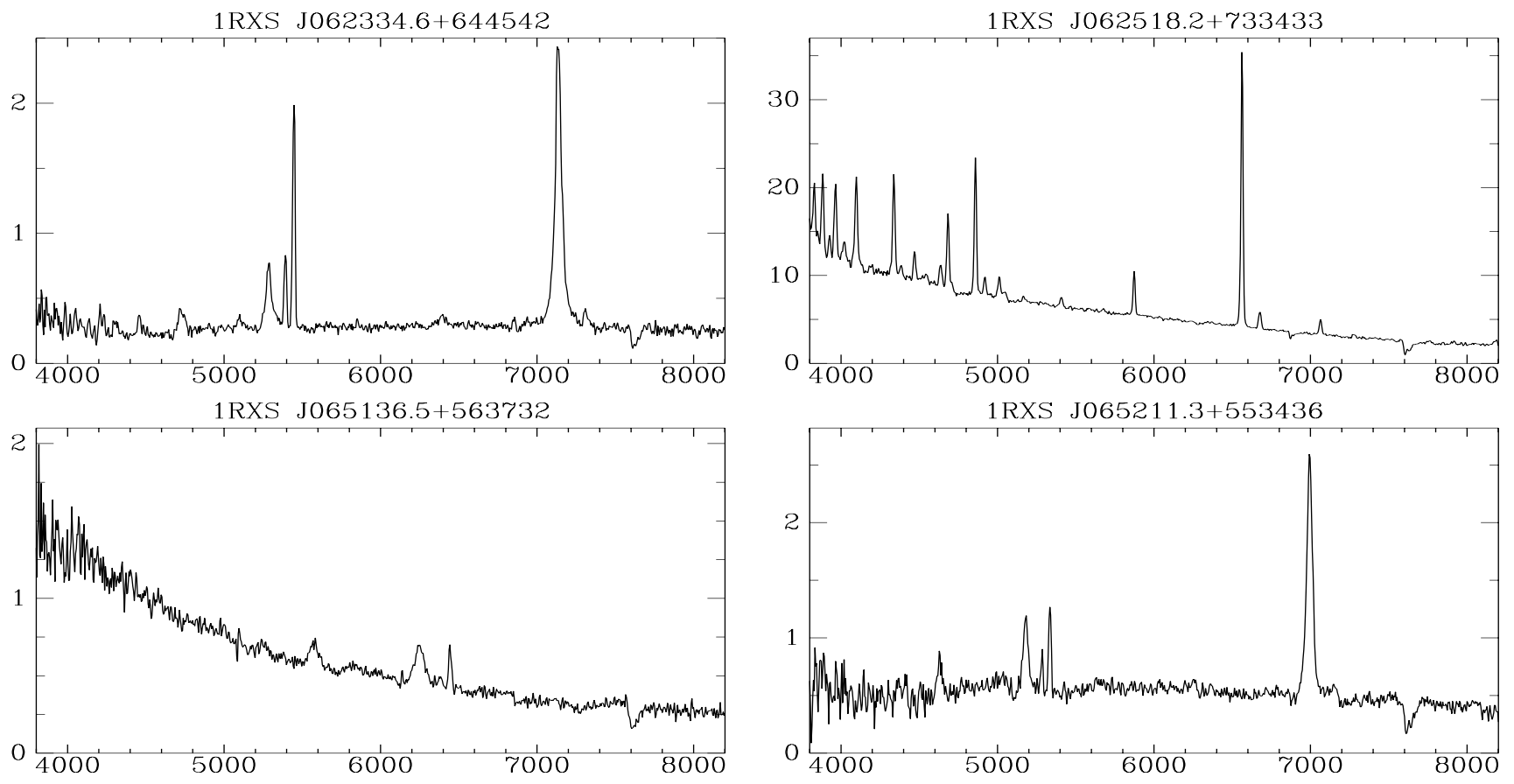

1RXS J065211.3+553436
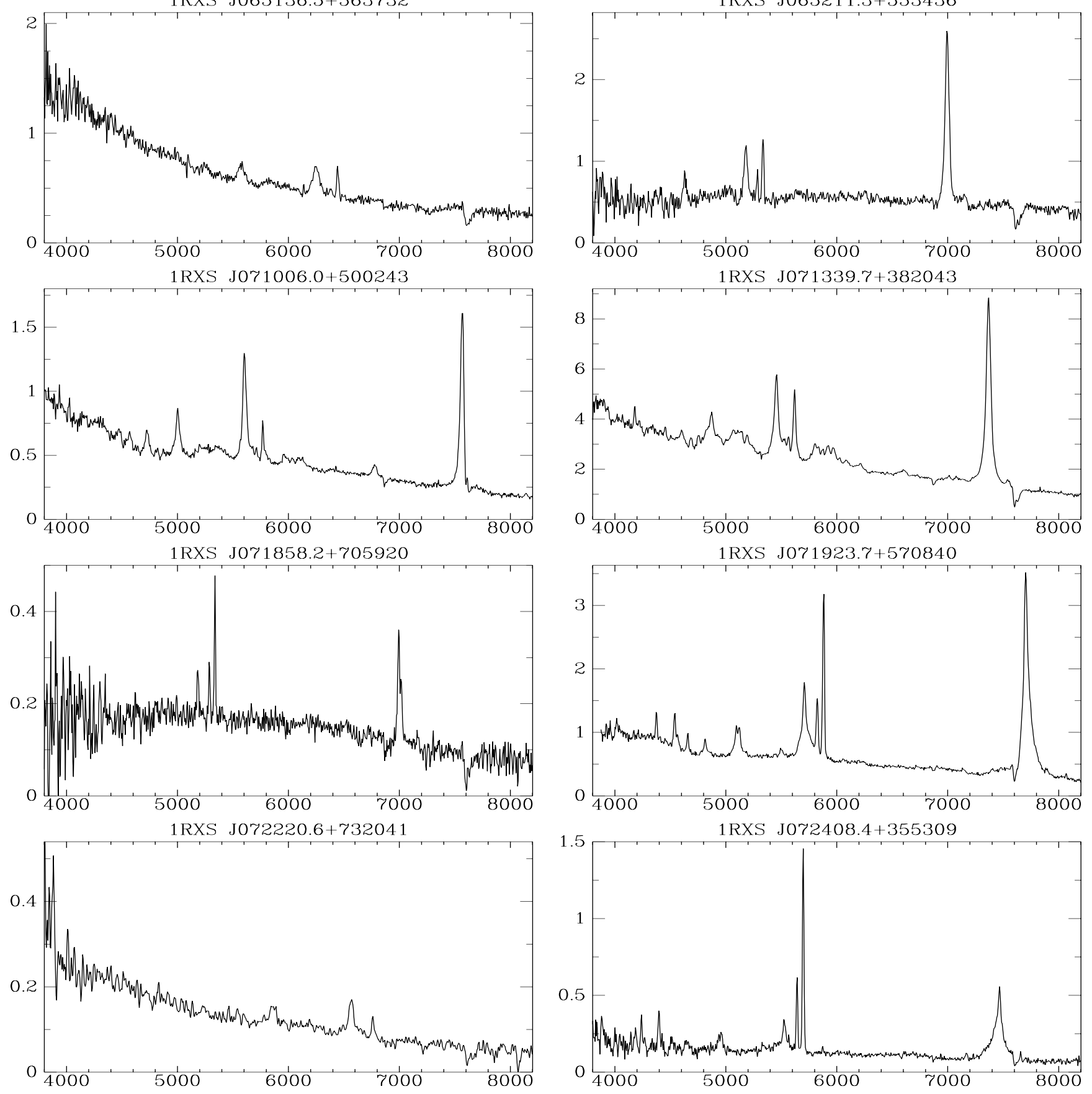

Fig. 2. continued 

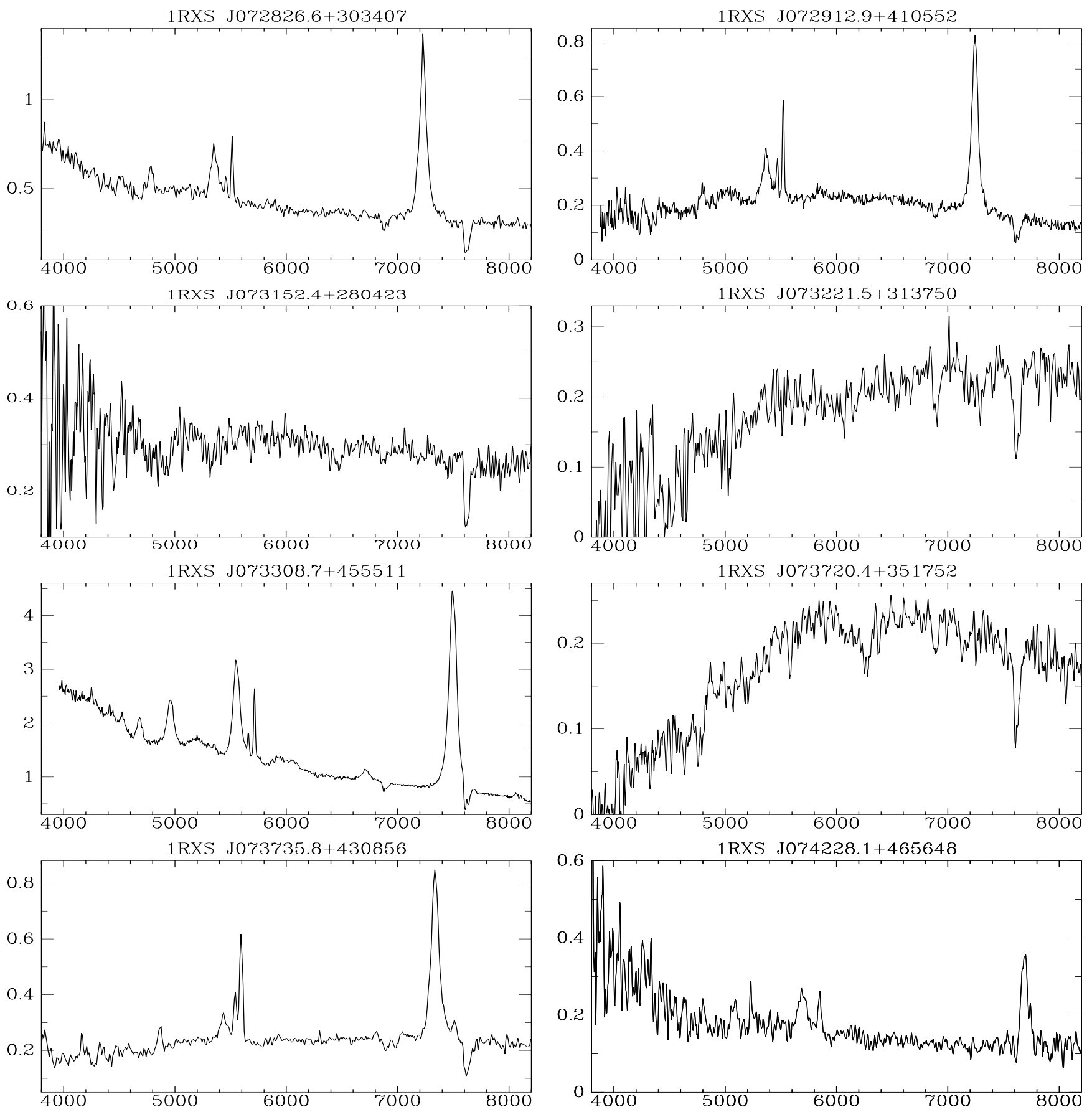
RXS J074311.3+742926

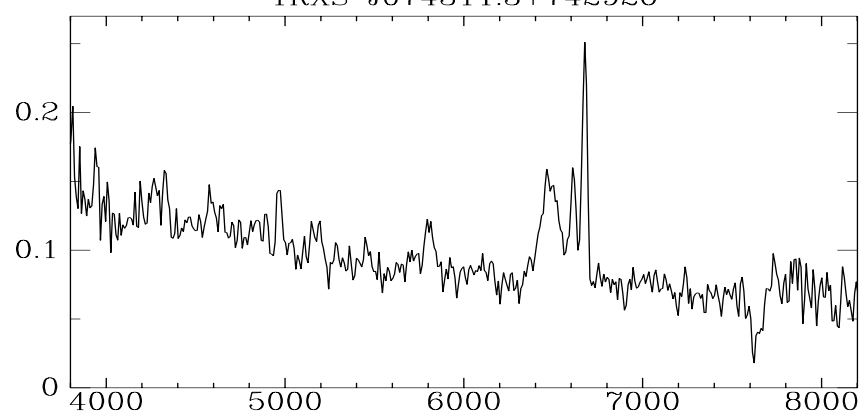
1 RXS J074759.2+205229

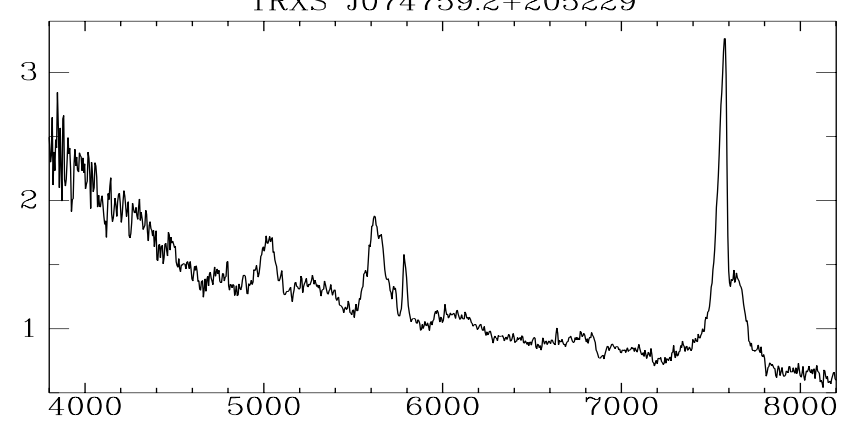

Fig. 2. continued 

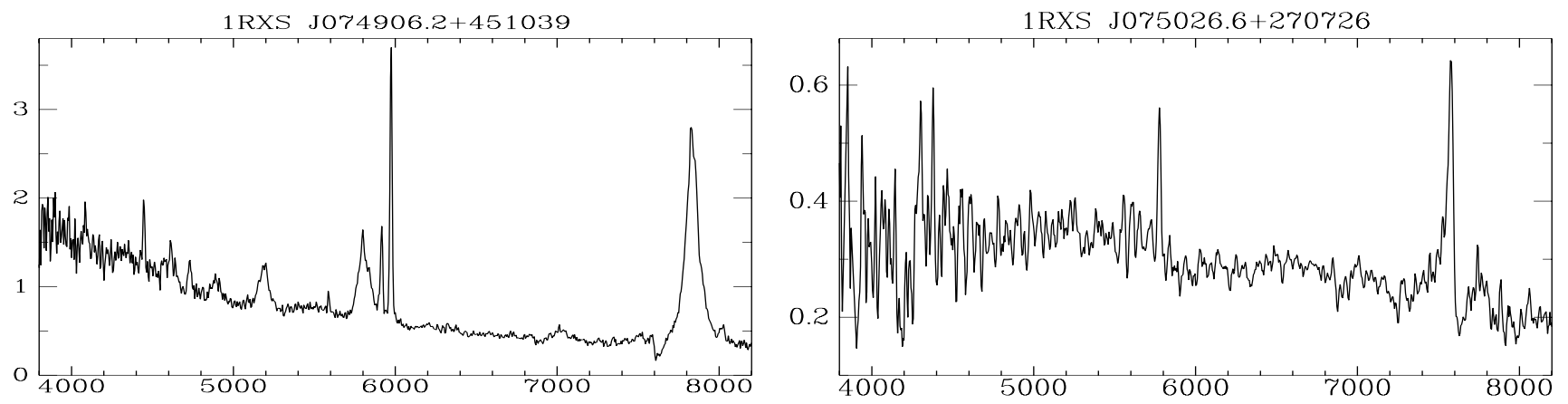

1 RXS J075039.5+624205
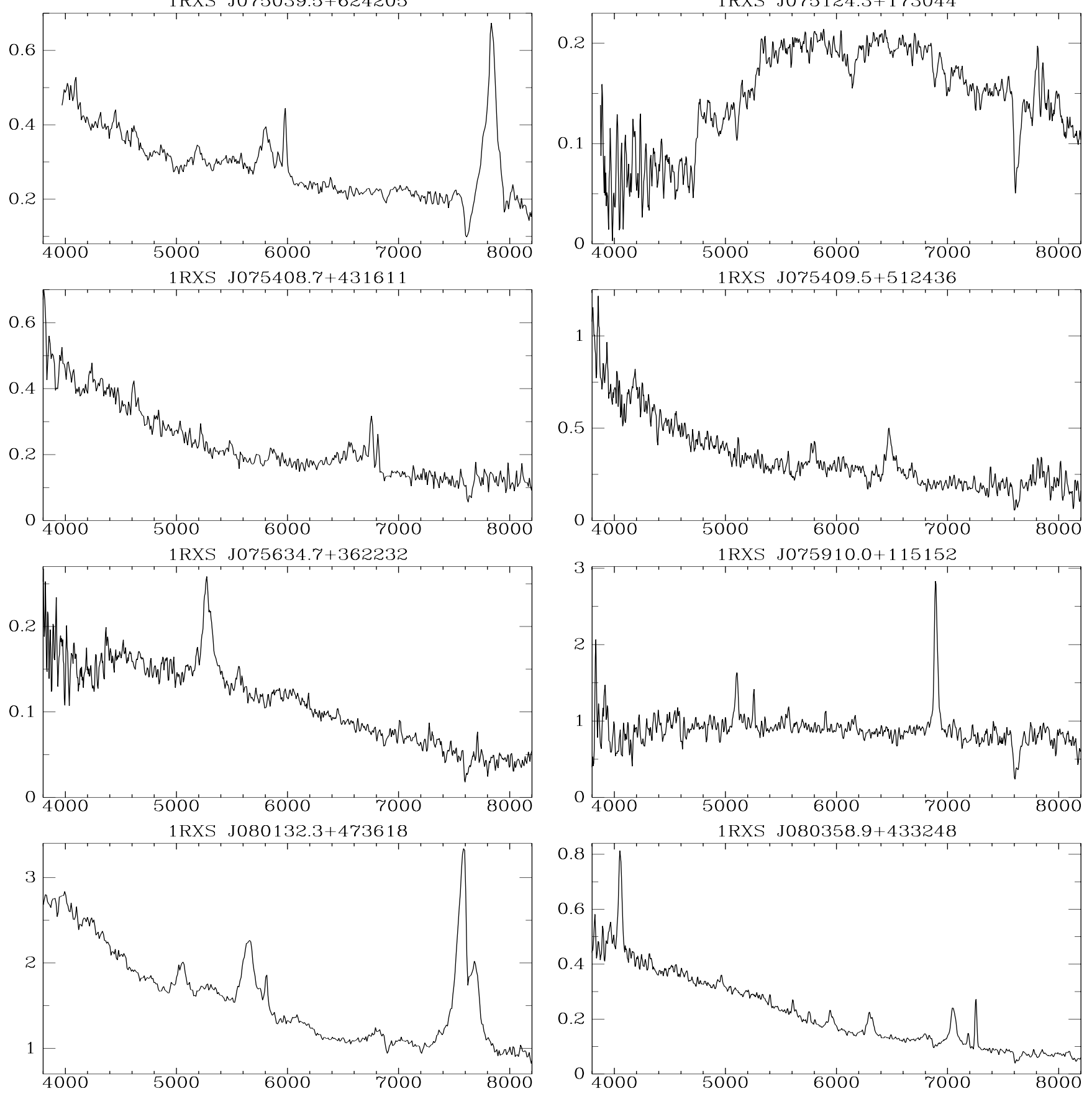

Fig. 2. continued 

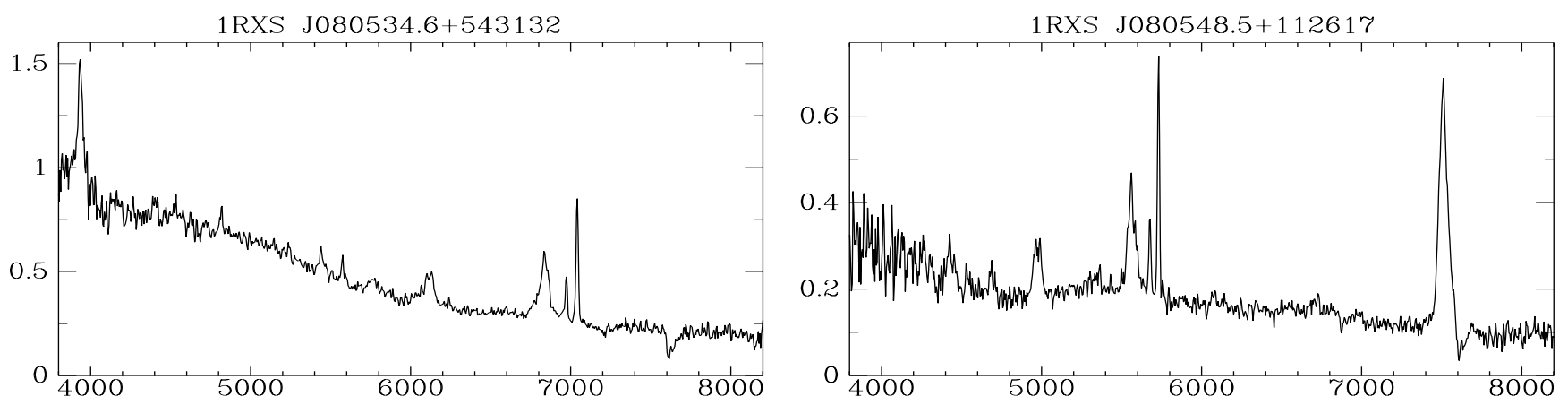
1RXS J080625.5+593105
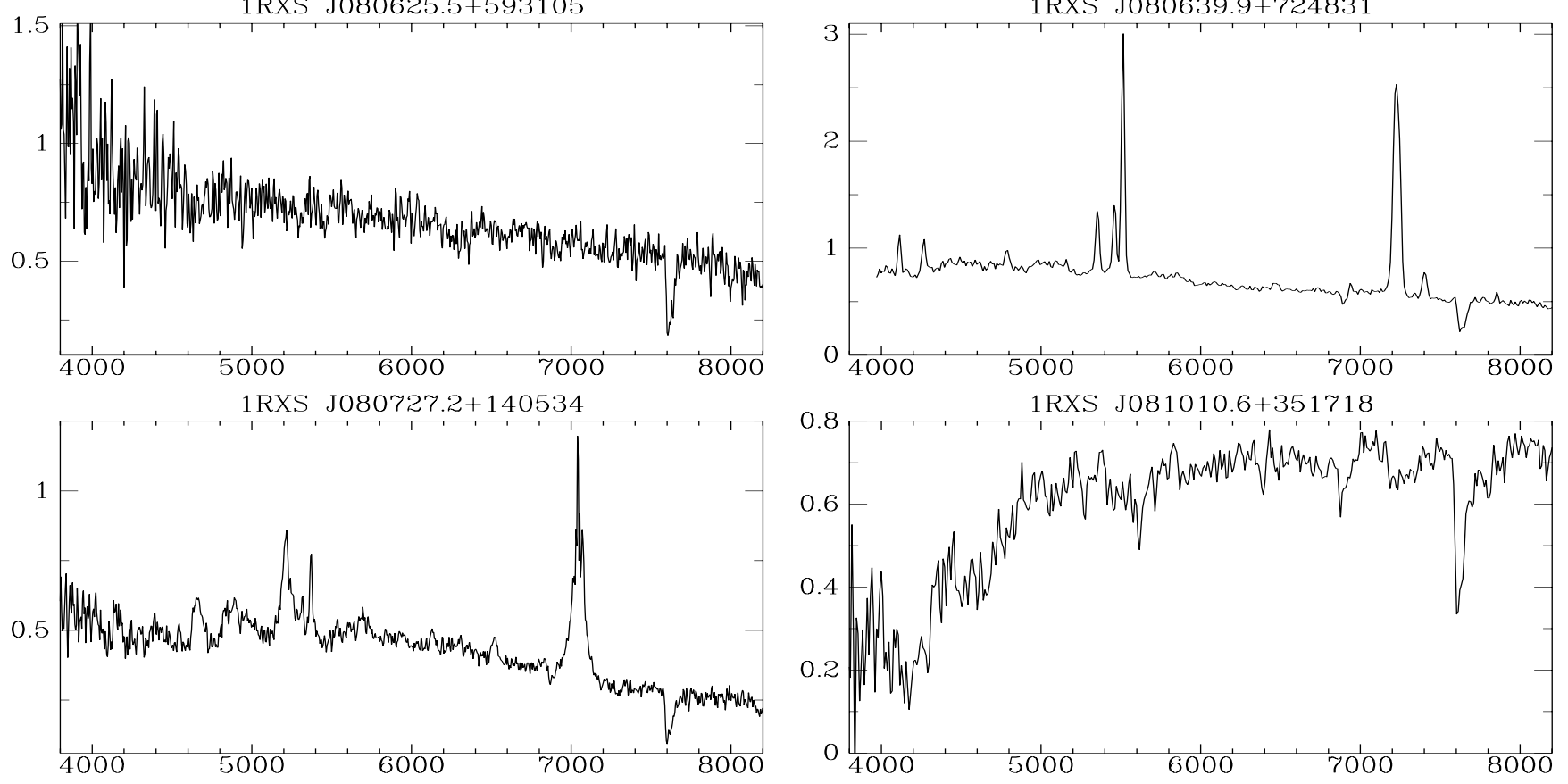
1RXS J081021.3+42165?
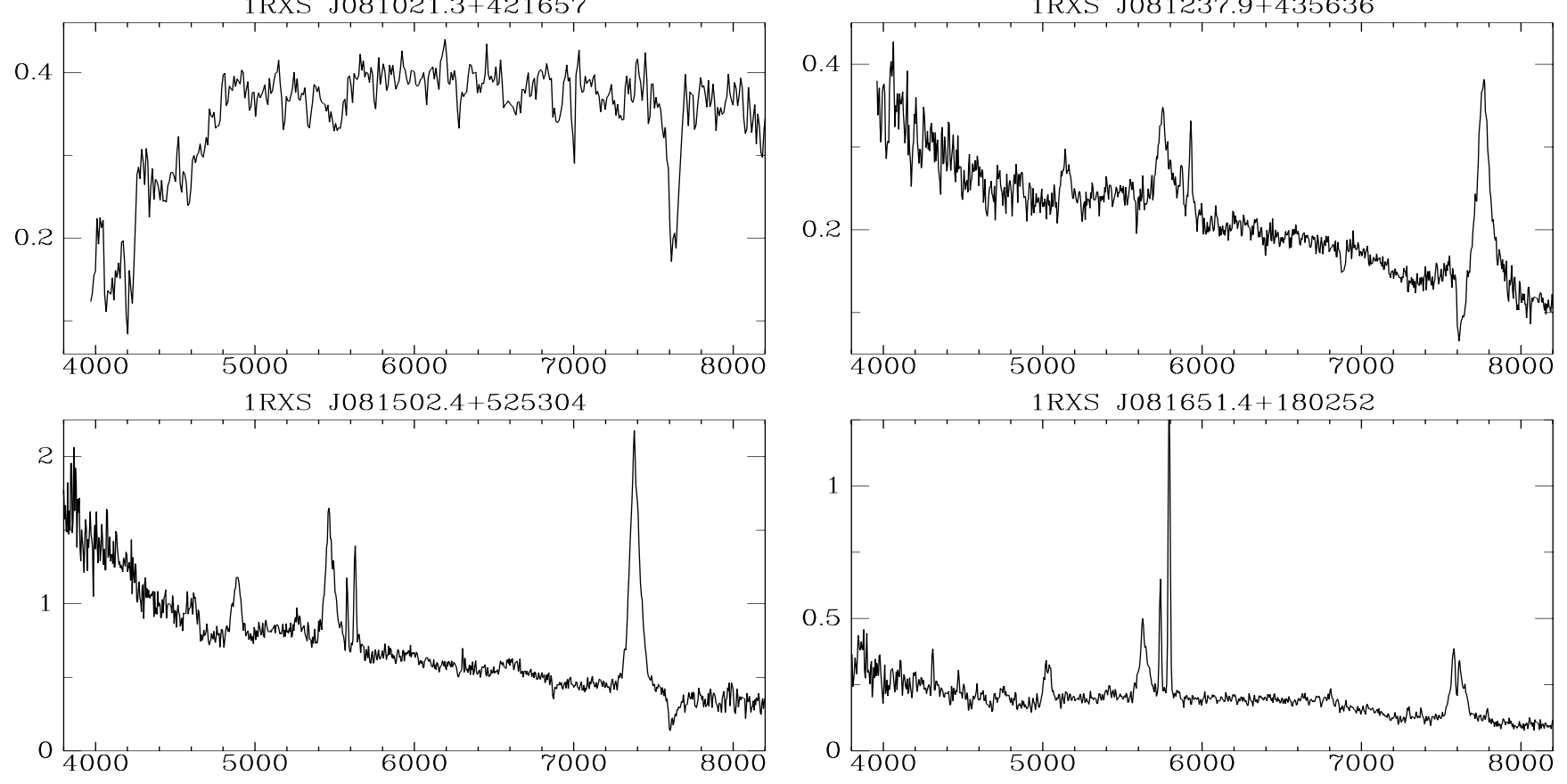

Fig. 2. continued 

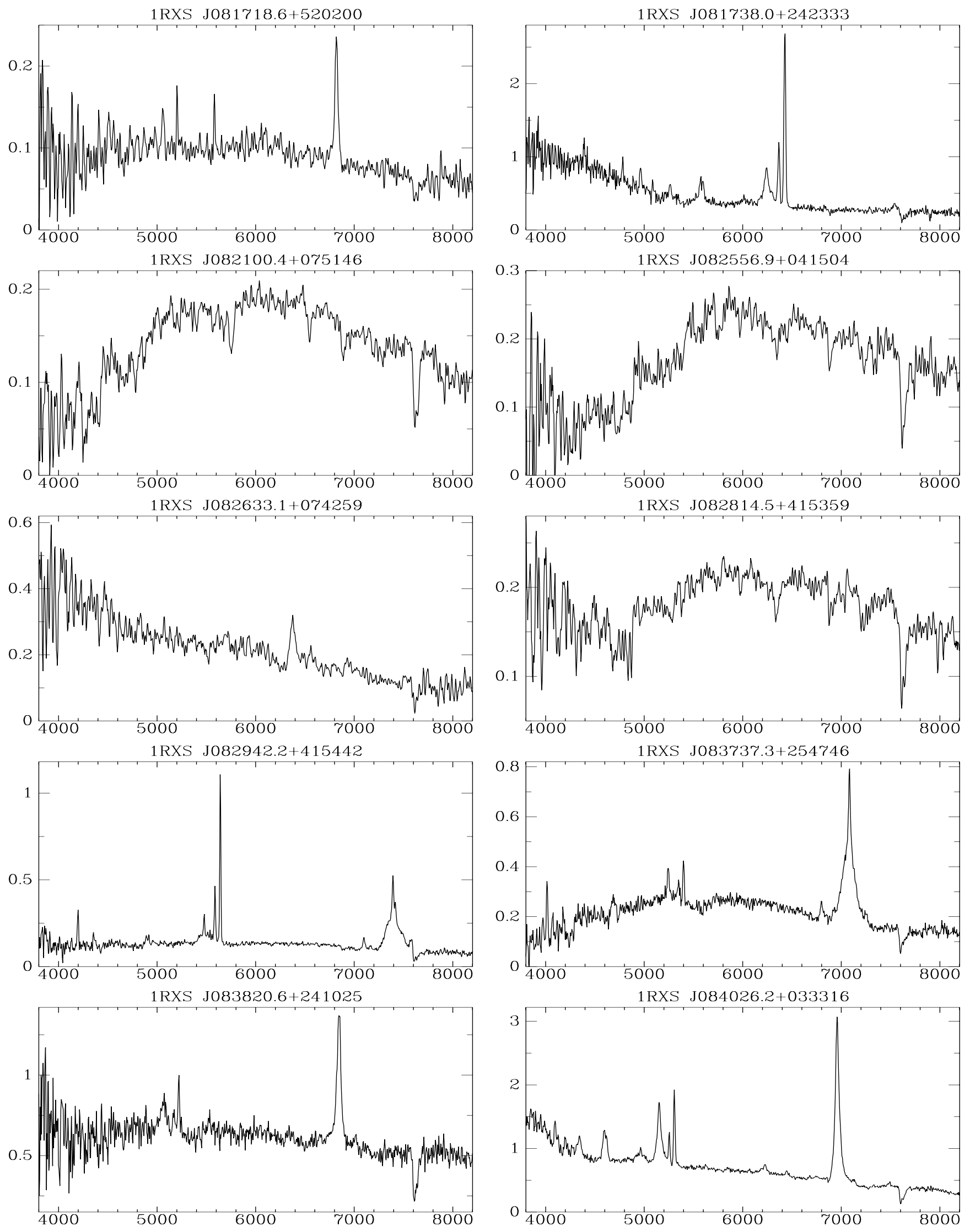

Fig. 2. continued 

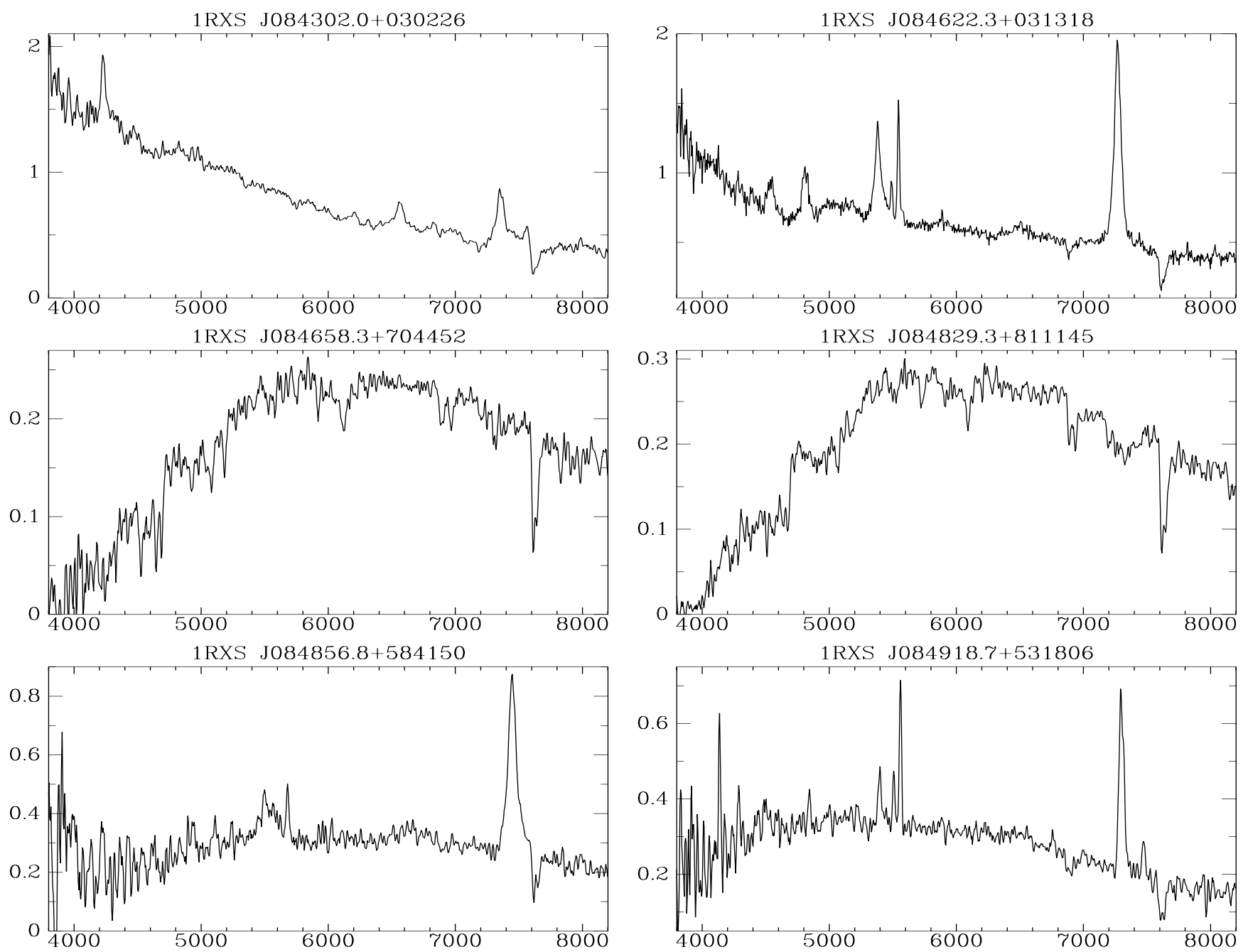

1 RXS J085233.3+422539
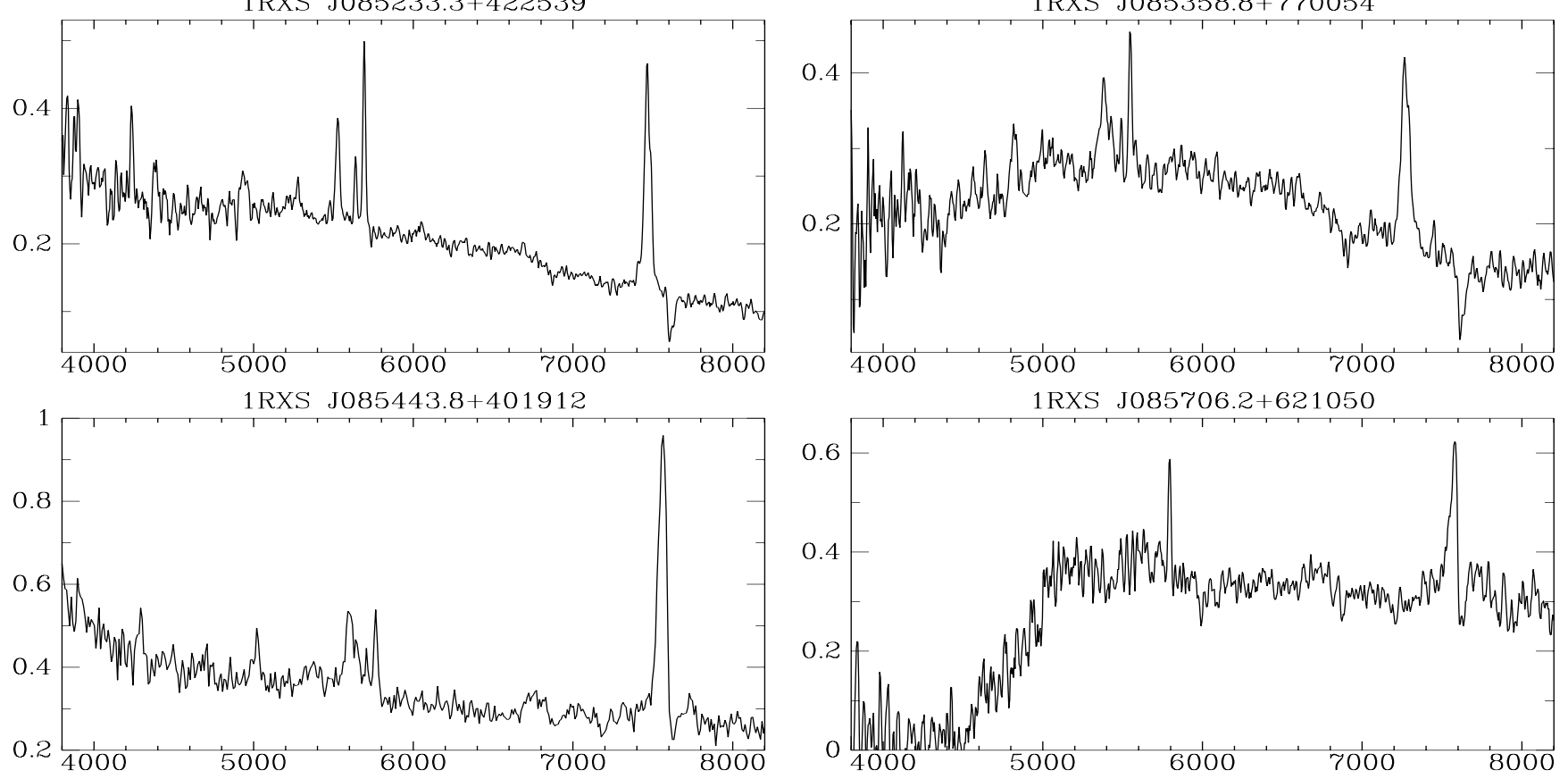

Fig. 2. continued 

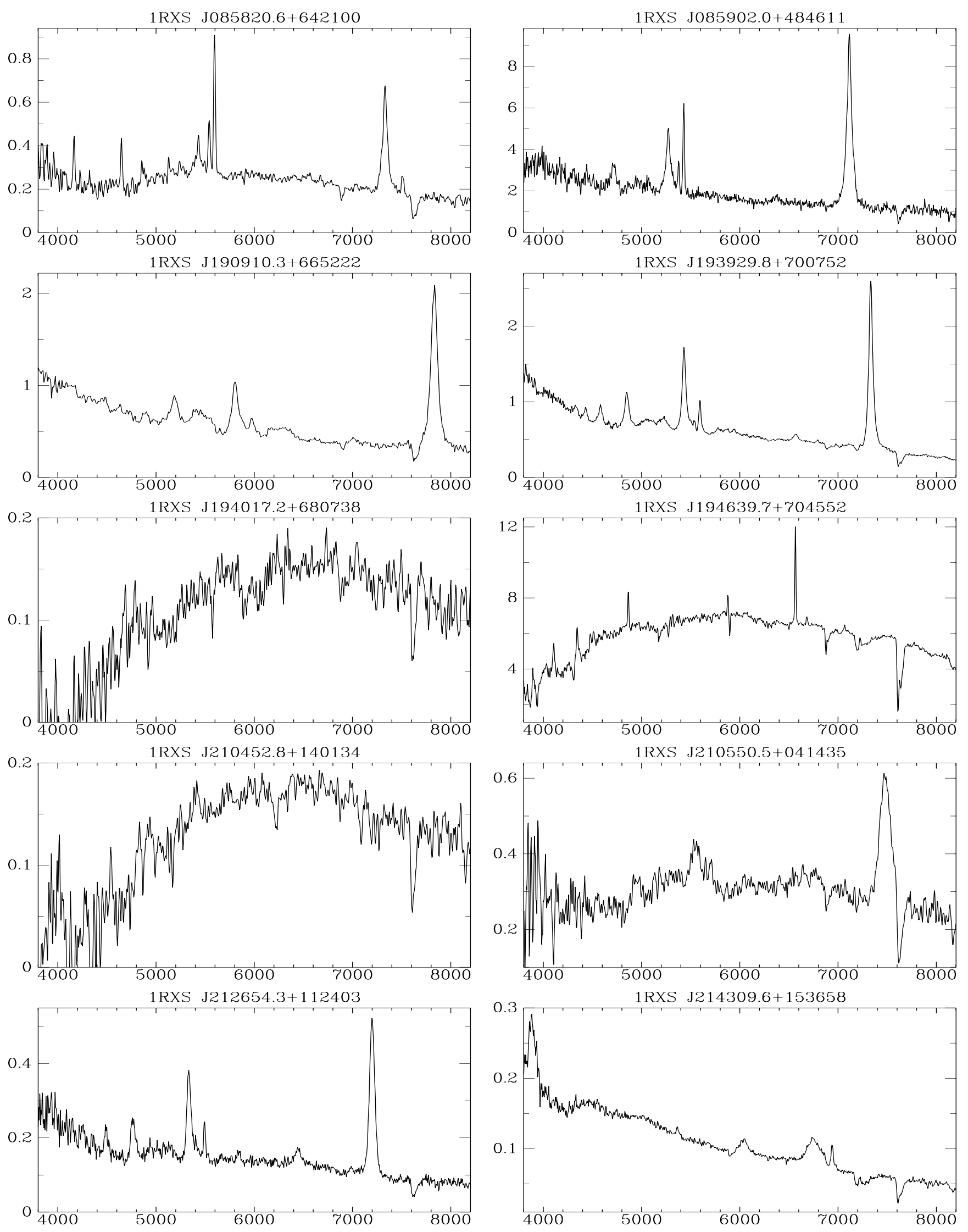

Fig. 2. continued 

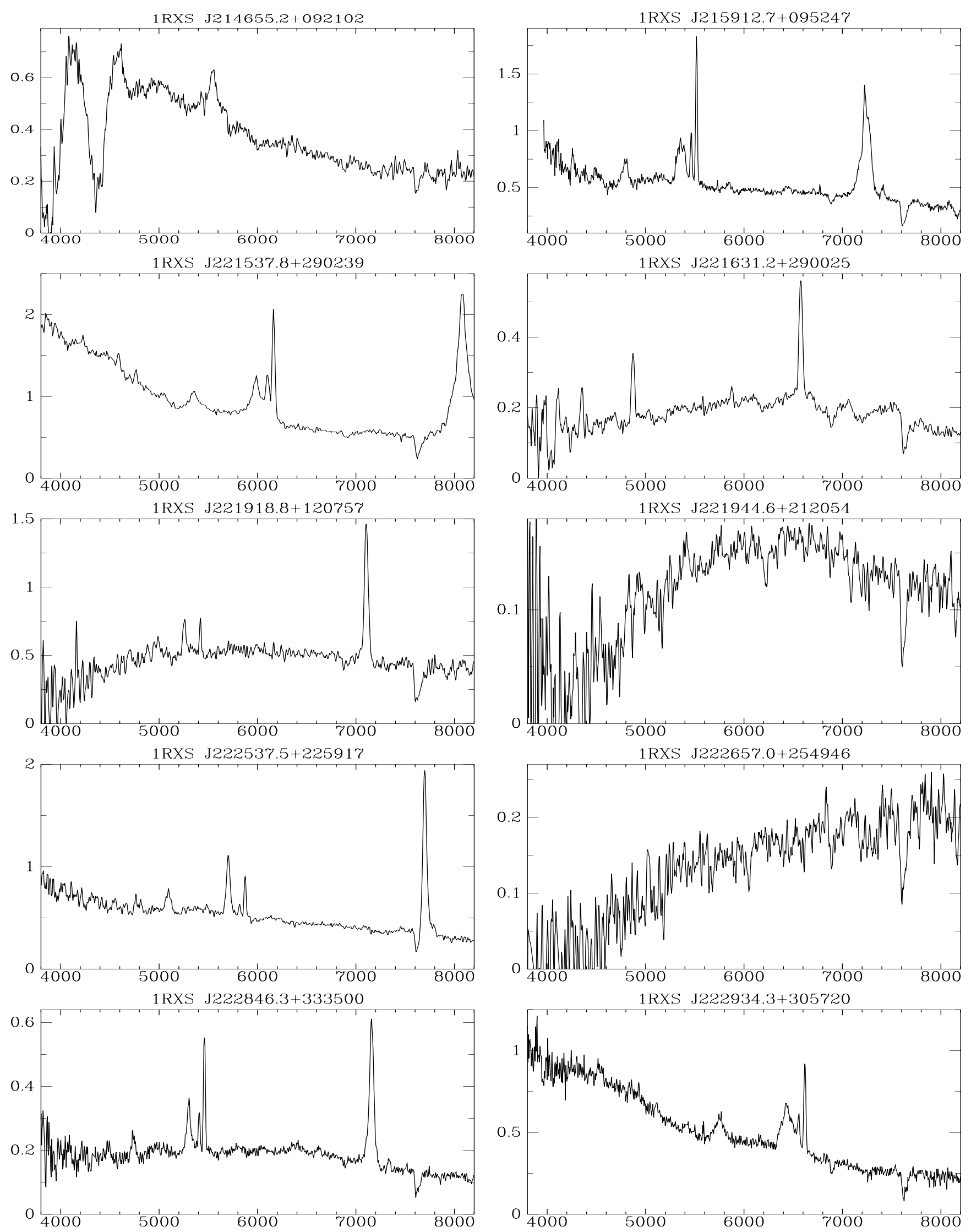

Fig. 2. continued 

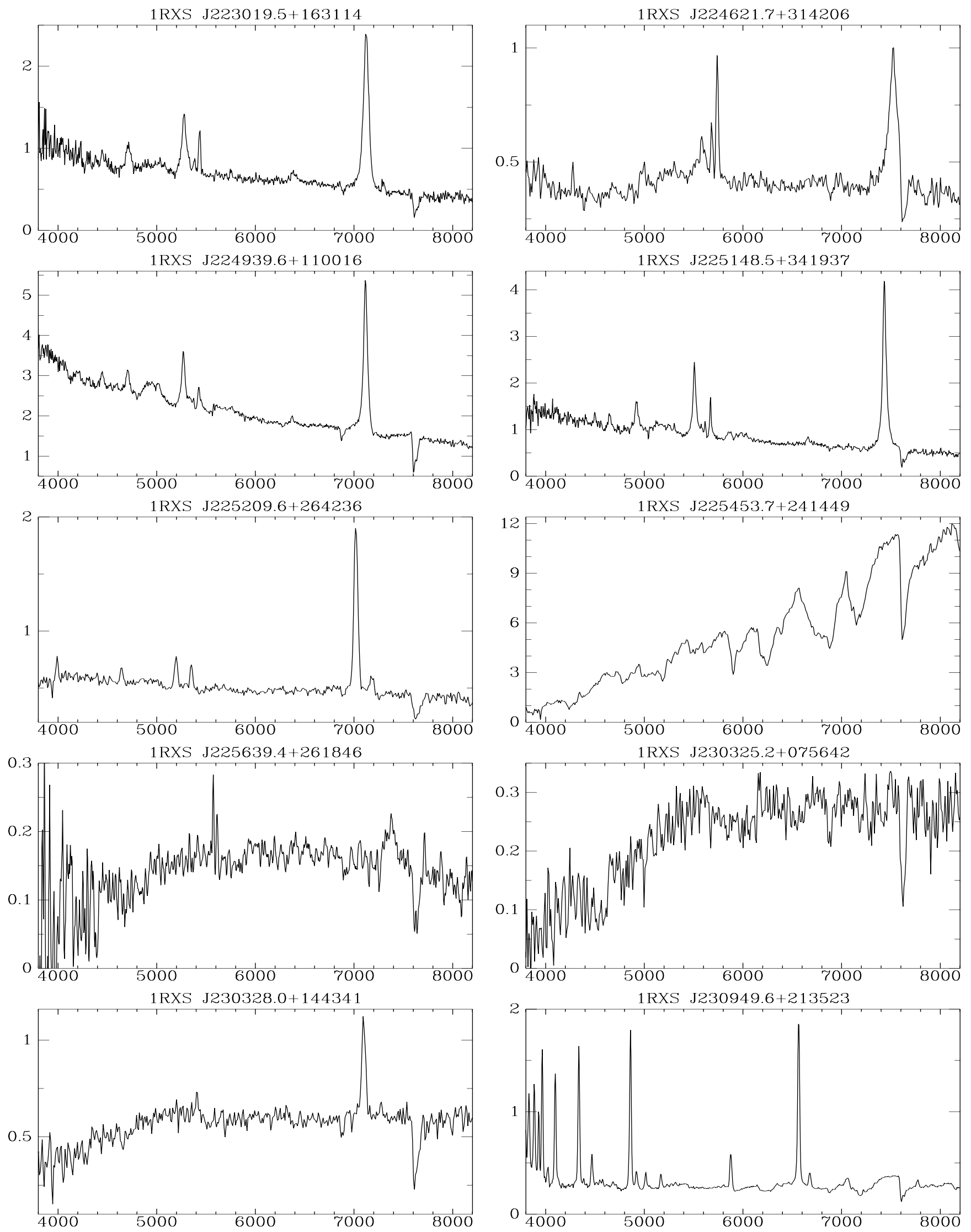

Fig. 2. continued 

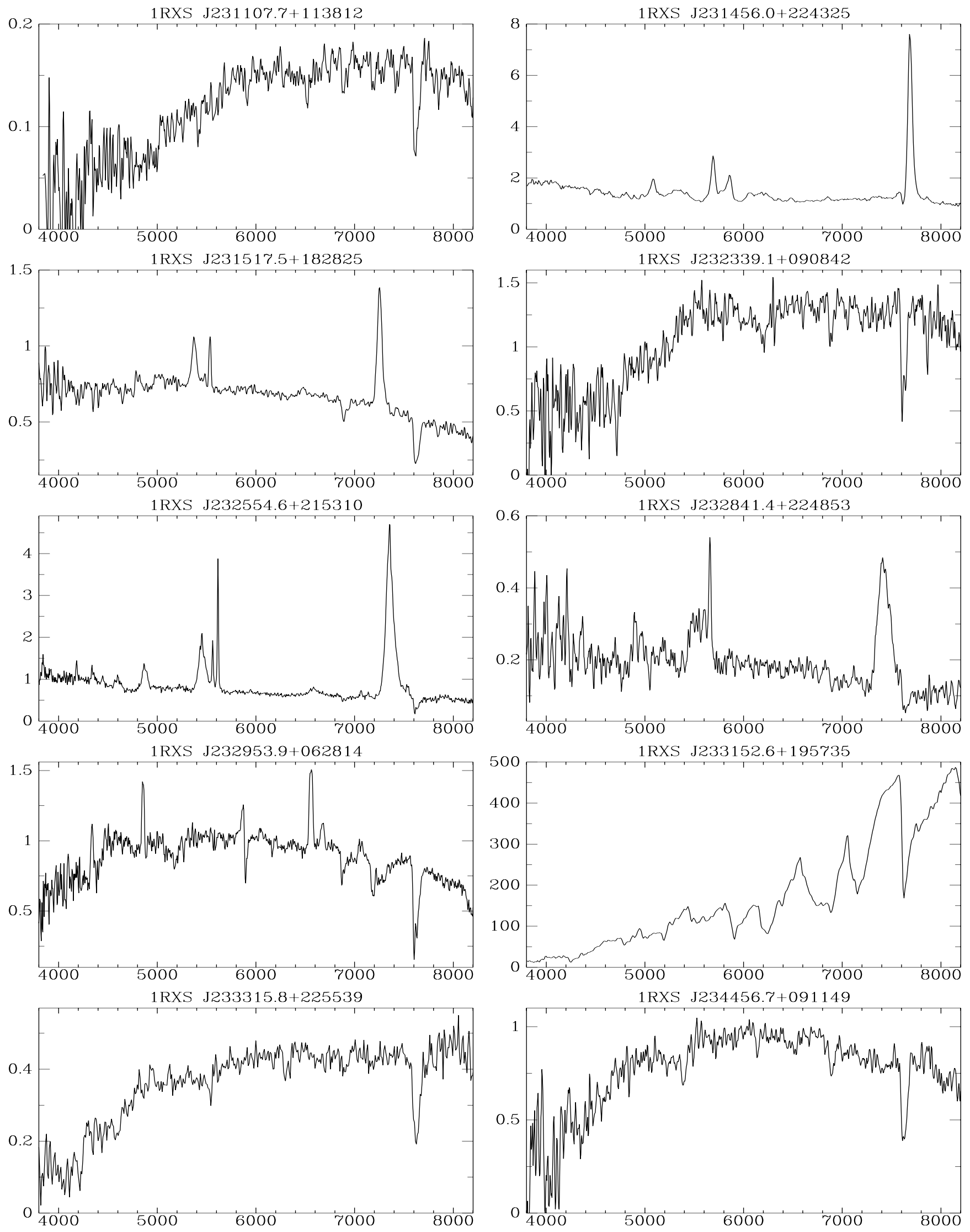

Fig. 2. continued 

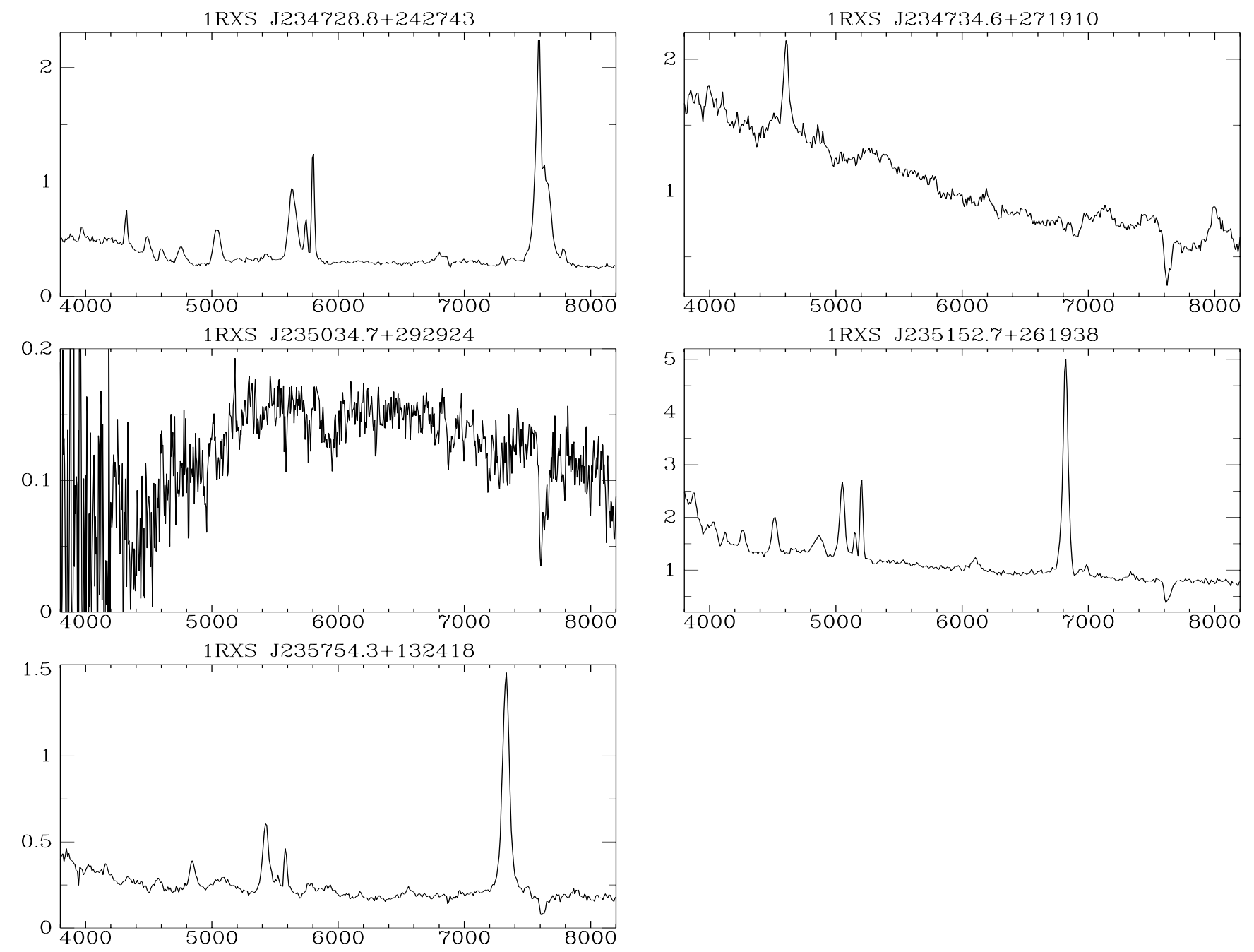

Fig. 2. continued

Table 1. X-ray parameters for four BL Lac candidates

\begin{tabular}{lllllcccc}
\hline \hline $\begin{array}{l}\text { ROSAT name } \\
\text { 1RXS J }\end{array}$ & $z$ & $\mathrm{CR}$ & $\Gamma_{\text {Gal }}^{\dagger}$ & $\begin{array}{c}f_{\mathrm{X}} \\
\mathrm{e}-12\end{array}$ & $\begin{array}{c}L_{\mathrm{X}} \\
\mathrm{erg} \mathrm{s}^{-1}\end{array}$ & $\begin{array}{c}f_{\mathrm{r}} \\
\mathrm{mJy}\end{array}$ & $\alpha_{\text {ox }}$ & $\alpha_{\text {ro }}$ \\
\hline $073720.4+351752$ & 0.213 & 0.090 & 1.46 & 1.69 & $3.6410^{45}$ & 27.2 & 1.19 & 0.43 \\
$082814.5+415359$ & 0.223 & 0.089 & 1.60 & 1.69 & $4.1310^{44}$ & 91.0 & 1.19 & 0.52 \\
$084829.3+811145$ & 0.175 & 0.118 & 3.38 & 3.42 & $6.6710^{44}$ & 12.9 & 1.11 & 0.36 \\
$221944.6+212054$ & 0.20 & 0.080 & 2.65 & 2.83 & $6.6410^{44}$ & 177.6 & 1.43 & 0.43 \\
\hline
\end{tabular}

redshift of the object by assuming spectral slopes within each band of $\alpha_{\mathrm{r}}=-0.7 ; \alpha_{\mathrm{x}}=-1$ and $\alpha_{\mathrm{o}}=-1.4$ (Ghisellini et al. 1986).

X-ray photon index for a power law fit with Galactic absorption.

\section{Results}

At present over $93 \%$ of the 165 X-ray sources have been successfully identified in the following classes: 115 emission line AGN (QSOs and Seyferts), 2 BL Lac objects and
4 BL Lac candidates, 22 clusters of galaxies, 12 Galactic stars. The other $10 \mathrm{X}$-ray sources remain unidentified because the proposed stellar counterparts are too faint to be the likely X-ray emitters. This represents a success rate of about $73 \%$ for detecting AGN using our selection criteria.

The final results of the identification are listed in Table 2 containing the following information in Cols. 12 to 14:

Column (12): Identification classification of the counterpart based on the criteria described in Sect. 4. 
Table 2. X-ray identification information

\begin{tabular}{|c|c|c|c|c|c|c|c|c|c|c|c|c|c|}
\hline $\begin{array}{l}\text { ROSAT name } \\
1 \mathrm{RXS} J\end{array}$ & $\begin{array}{l}\text { RA } \\
(2000)\end{array}$ & $\begin{array}{l}\text { Dec } \\
(2000)\end{array}$ & $\begin{array}{l}\text { P.E } \\
\left({ }^{\prime \prime}\right)\end{array}$ & $\begin{array}{r}\mathrm{D} \\
\left({ }^{\prime \prime}\right)\end{array}$ & $\begin{array}{c}\mathrm{CR} \\
\left(\operatorname{cts~s}^{-1}\right)\end{array}$ & $H R 1$ & $H R 2$ & $\begin{array}{l}\text { ext } \\
\left({ }^{\prime \prime}\right)\end{array}$ & $R$ & $B$ & ID & $z$ & $M_{B}$ \\
\hline (1) & $(2)$ & $(3)$ & (4) & (5) & (6) & (7) & (8) & (9) & $(10)$ & (11) & $(12)$ & (13) & (14) \\
\hline $000011.9+052318$ & 000011.76 & 052317.3 & 7 & 2.2 & 0.264 & 0.24 & 0.00 & 0 & 15.5 & 16.4 & Sey & 0.040 & -20.5 \\
\hline $000055.5+172346$ & 000055.98 & 172339.0 & 12 & 9.8 & 0.065 & 0.10 & -0.13 & 14 & 16.5 & 17.2 & QSO & 0.215 & -23.4 \\
\hline $000550.0+102249$ & 000549.54 & 102237.2 & 9 & 13.6 & 0.073 & 0.85 & -0.08 & 0 & 15.9 & 16.7 & Sey & 0.095 & -22.1 \\
\hline $000620.3+105207$ & 000620.33 & 105151.0 & 27 & 16.0 & 0.135 & 0.91 & 0.72 & 100 & 14.9 & 18.0 & CL & 0.167 & \\
\hline $000915.0+243758$ & 000915.21 & 243739.3 & 20 & 18.9 & 0.118 & 0.45 & -0.30 & 21 & 16.4 & 17.6 & Sey & 0.118 & -21.7 \\
\hline $000953.0+371906$ & 000952.42 & 371910.1 & 13 & 8.0 & 0.082 & 0.70 & -0.20 & 0 & 15.5 & 16.6 & QSO & 0.154 & -23.3 \\
\hline $001121.2+373919$ & 001121.02 & 373923.0 & 11 & 4.5 & 0.126 & 0.90 & 0.21 & 21 & 15.2 & 16.5 & Sey & 0.104 & -22.5 \\
\hline $001452.7+270549$ & 001453.04 & 270541.5 & 11 & 8.8 & 0.171 & 0.18 & 0.15 & 7 & 15.6 & 16.4 & Sey & 0.086 & -22.2 \\
\hline $002007.2+324423$ & 002007.31 & 324409.9 & 12 & 13.2 & 0.083 & 0.54 & 0.09 & 0 & 15.6 & 17.0 & Sey & 0.082 & -21.5 \\
\hline \multirow[t]{3}{*}{$002136.8+280305$} & 002136.32 & 280310.9 & 23 & 8.7 & 0.160 & 1.00 & 0.17 & 78 & 13.4 & 16.3 & CL & 0.094 & \\
\hline & 002135.42 & 280318.0 & & 22.4 & & & & & 14.9 & 18.3 & & & \\
\hline & 002136.87 & 280301.9 & & 3.2 & & & & & 11.7 & 15.1 & & & \\
\hline $002433.0+331237$ & 002432.89 & 331224.6 & 27 & 12.5 & 0.097 & 0.74 & 0.11 & 72 & 15.0 & 19.0 & CL & 0.225 & \\
\hline $002445.3+082047$ & 002445.75 & 082056.6 & 20 & 11.7 & 0.060 & 0.04 & -0.76 & 0 & 15.8 & 17.2 & Sey & 0.067 & -20.8 \\
\hline $002811.6+310342$ & 002810.76 & 310347.8 & 9 & 12.3 & 0.168 & 0.64 & 0.05 & 14 & 15.3 & 15.8 & QSO & 0.500 & -26.7 \\
\hline $002902.7+195717$ & 002902.95 & 195709.9 & 10 & 7.9 & 0.082 & 0.68 & 0.34 & 0 & 16.5 & 17.5 & QSO & 0.264 & -23.6 \\
\hline $003121.2+301558$ & 003122.02 & 301602.1 & 14 & 11.4 & 0.050 & 0.34 & -0.01 & 0 & 16.3 & 18.2 & Sey & 0.200 & -22.7 \\
\hline $003221.1+242359$ & 003221.04 & 242359.3 & 13 & 0.9 & 0.090 & 0.42 & -0.26 & 0 & 15.4 & 17.3 & Sey & 0.066 & -20.7 \\
\hline $003952.8+313228$ & 003951.92 & 313231.7 & 10 & 11.8 & 0.135 & -1.00 & 0.00 & 0 & 14.6 & 14.7 & WD & & \\
\hline $004459.4+192143$ & 004459.13 & 192140.8 & 9 & 4.4 & 0.166 & -0.20 & -0.22 & 0 & 16.1 & 16.4 & QSO & 0.179 & -23.8 \\
\hline $005034.2+044152$ & 005033.77 & 044148.8 & 11 & 7.2 & 0.052 & -0.08 & 0.29 & 0 & 15.7 & 17.2 & Sey & 0.097 & -21.7 \\
\hline $005050.6+353645$ & 005050.79 & 353642.3 & 8 & 3.6 & 0.285 & 0.13 & -0.14 & 10 & 14.0 & 14.6 & QSO & 0.058 & -23.1 \\
\hline $005607.6+254802$ & 005607.70 & 254804.9 & 10 & 3.2 & 0.125 & 0.38 & 0.14 & 9 & 15.6 & 16.7 & QSO & 0.153 & -23.2 \\
\hline $011216.3+330243$ & 011217.13 & 330241.3 & 15 & 10.6 & 0.056 & 0.79 & 0.29 & 0 & 16.4 & 18.8 & & & \\
\hline $011655.1+254937$ & 011654.07 & 254927.8 & 12 & 16.7 & 0.079 & 0.95 & 0.51 & 13 & 15.3 & 16.7 & Sey & 0.099 & -22.2 \\
\hline $011745.5+363718$ & 011745.64 & 363714.1 & 8 & 4.3 & 0.213 & 0.78 & 0.08 & 8 & 15.3 & 16.3 & Sey & 0.107 & -22.8 \\
\hline $011747.9+361042$ & 011749.45 & 361026.2 & 24 & 24.5 & 0.056 & 0.10 & 0.53 & 0 & 16.5 & 18.7 & & & \\
\hline $011848.2+383626$ & 011849.48 & 383619.6 & 15 & 16.3 & 0.059 & 0.00 & -0.46 & 19 & 16.4 & 16.8 & QSO & 0.216 & -23.8 \\
\hline $012028.0+384918$ & 012026.39 & 384915.5 & 28 & 19.0 & 0.089 & 1.00 & 0.29 & 62 & 16.4 & 17.4 & & & \\
\hline $012556.6+351039$ & 012555.96 & 351036.9 & 13 & 8.1 & 0.059 & 0.68 & 0.38 & 0 & 15.6 & 17.3 & QSO & 0.312 & -24.2 \\
\hline $012923.0+223100$ & 012922.48 & 223110.6 & 12 & 12.8 & 0.058 & 0.65 & 0.16 & 0 & 16.4 & 17.0 & QSO & 0.231 & -23.8 \\
\hline $013632.9+390556$ & 013632.60 & 390559.1 & 7 & 4.7 & 0.750 & 0.76 & 0.22 & 8 & 14.9 & 15.9 & $\mathrm{BL}$ & & \\
\hline $013738.5+852414$ & 013737.21 & 852410.7 & 8 & 3.7 & 0.061 & 0.77 & 0.41 & 0 & 16.3 & 17.0 & QSO & 0.499 & -25.5 \\
\hline $013853.1+252325$ & 013852.82 & 252325.2 & 9 & 3.8 & 0.532 & -0.93 & 1.00 & 28 & 15.9 & 15.5 & WD & & \\
\hline $014023.0+240255$ & 014022.91 & 240251.3 & 13 & 3.9 & 0.140 & 0.79 & -0.05 & 27 & 15.9 & 17.0 & Sey & 0.072 & -21.2 \\
\hline $014025.2+382400$ & 014024.35 & 382409.3 & 9 & 13.6 & 0.212 & 0.39 & 0.00 & 7 & 16.1 & 16.9 & Sey & 0.067 & -21.1 \\
\hline $014732.3+345358$ & 014732.37 & 345354.4 & 8 & 3.7 & 0.182 & -0.30 & 0.23 & 0 & 14.2 & 16.9 & M & & \\
\hline $015505.4+335452$ & 015506.26 & 335512.0 & 20 & 22.7 & 0.222 & 1.00 & 0.33 & 77 & 16.2 & 18.8 & CL & 0.092 & \\
\hline
\end{tabular}


Table 2. continued

\begin{tabular}{|c|c|c|c|c|c|c|c|c|c|c|c|c|c|}
\hline $\begin{array}{l}\text { ROSAT name } \\
\text { 1RXS J } \\
(1)\end{array}$ & $\begin{array}{l}\text { RA } \\
(2000) \\
(2) \\
\end{array}$ & $\begin{array}{l}\text { Dec } \\
(2000) \\
(3) \\
\end{array}$ & $\begin{array}{l}\text { P.E } \\
\left({ }^{\prime \prime}\right) \\
(4)\end{array}$ & $\begin{array}{r}\mathrm{D} \\
\left({ }^{\prime \prime}\right) \\
(5)\end{array}$ & $\begin{array}{l}\mathrm{CR} \\
\left.\mathrm{ts} \mathrm{s}^{-1}\right) \\
(6)\end{array}$ & (7) & $H R 2$ & $\begin{array}{l}\text { ext } \\
\left({ }^{\prime \prime}\right) \\
(9)\end{array}$ & $(10)$ & $(11)$ & $(12)$ & $(13)$ & $(14)$ \\
\hline $015536.7+311525$ & 15536.05 & 311518.0 & 14 & 10.9 & 0.055 & 0.18 & -0.34 & 0 & 15.9 & 17.3 & Sey & 0.135 & -22.3 \\
\hline $015546.4+071902$ & 15546.39 & 71903.8 & 14 & 1.8 & 0.076 & 0.71 & 0.09 & 15 & 15.4 & 17.7 & y & .069 & -20.4 \\
\hline $015555.6+040620$ & 1555528 & 040619.3 & 10 & 4.8 & 0.094 & 0.40 & 0.42 & 13 & 15.9 & 17.6 & oy & .136 & -22.0 \\
\hline $015621.6+241838$ & 015621.37 & 241837.4 & 11 & 3.2 & 0.065 & 0.90 & -0.07 & 0 & 16.3 & 17.0 & y & .155 & -22.9 \\
\hline $020348.7+295921$ & 020348.66 & 95926.3 & 7 & 5.3 & 0.539 & -0.96 & -1.00 & 5 & 16.0 & 15.5 & ry & & \\
\hline \multirow[t]{3}{*}{$022851.6+335949$} & 022853.21 & 335940.7 & 37 & 21.7 & 0.080 & 0.93 & 0.22 & 81 & 15.5 & 16.1 & & & \\
\hline & 022853.70 & 340020.2 & 37 & 40.7 & 0.080 & 0.93 & 0.22 & 81 & 16.0 & 20.2 & CL & 0.19 & \\
\hline & 022851.33 & 335910.6 & 37 & 38.5 & 0.080 & 0.93 & 0.22 & 81 & 16.1 & 17.8 & & & \\
\hline $023601.9+$ & 023601.37 & 082127.2 & 9 & 8.5 & 0.092 & 0.23 & 0.20 & 5 & 16.1 & 17.3 & & & -23.5 \\
\hline $023739.9+181945$ & 023740.49 & 181941.8 & 12 & 9.0 & 0.099 & 1.00 & 0.55 & 0 & 15.9 & 16.4 & QSO & 168 & -23.7 \\
\hline $024214.5+074442$ & 024214.20 & 4453.3 & 16 & 1 & 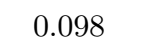 & 74 & 7 & 25 & 16.3 & 16.5 & & 1 & -25.8 \\
\hline $033522.6+190739$ & 33522.59 & 190728.5 & 8 & 10.5 & 0.281 & 0.98 & 0.44 & 9 & 15.8 & 17.1 & $\mathrm{O}$ & 89 & -23.2 \\
\hline $034308.0+185836$ & 3420 & 858. & 12 & 11.3 & 0183 & 00 & 42 & 5 & 4 & 16.7 & & 10 & -22.4 \\
\hline $034314.1+102941$ & 34314.26 & 102939.3 & 9 & 2.9 & 0.210 & 0.95 & 0.01 & 0 & 16.5 & 16.6 & & & \\
\hline $035147.6+103618$ & $\begin{array}{lll}03 & 51 & 8\end{array}$ & 1036075 & 9 & 10.9 & 0.095 & 0.90 & 0.00 & 9 & 16.5 & 17.6 & 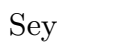 & 85 & -22.7 \\
\hline \multirow[t]{2}{*}{$035345.9+195825$} & 35346.24 & 195825.6 & 12 & 4.8 & 0.126 & 0.90 & 0.21 & 17 & 9.7 & 12.6 & & 29 & -23.7 \\
\hline & 035346.53 & 1958178 & & 11.4 & & & & & 16.1 & 17.9 & & & \\
\hline & 405 & 43.4 & 10 & 3.8 & 0.098 & 1.00 & 0.09 & 0 & 16.1 & 20.0 & & & -18.9 \\
\hline $041147.1+132417$ & 41 & 129 & 9 & 3.1 & 077 & 0.77 & 1 & 0 & 15.9 & 17.4 & $\mathrm{O}$ & 77 & -23.8 \\
\hline $044428.6+122113$ & 4442 & 122111.0 & 9 & 2.9 & 0.102 & 0.98 & 0.18 & 6 & 15.2 & 16.2 & & 0.089 & -22.5 \\
\hline $062334.6+644542$ & 23 & $\therefore$ & 9 & 6.7 & 0 & 87 & 0 & 9 & 16.0 & 17.2 & Sey & 86 & -21.4 \\
\hline $062518.2+733433$ & 06251 & 7334 & 9 & 10.2 & 0.180 & -0.32 & -0.02 & 14 & 14.5 & 14.8 & & & \\
\hline \multirow[t]{2}{*}{$065136.5+563732$} & 65136.66 & 563736.7 & 12 & 4.9 & 0.054 & 0.72 & -0.14 & 0 & 16.7 & 16.8 & 2SO & 0.286 & -24.5 \\
\hline & 065136.78 & 563720.7 & & 11.5 & & & & & 16.4 & 18.5 & & & \\
\hline $065211.3+553436$ & 5211.6 & 24 & 0 & 24 & 1 & 1 & 7 & 10 & 15.2 & 16.8 & & $\$ 4$ & -21.1 \\
\hline $071006.0+500243$ & 071006.75 & 500245.9 & 12 & 7.8 & 0.123 & 0.83 & 0.33 & 0 & 16.3 & 16.8 & & & -23.1 \\
\hline $071339.7+382043$ & 1340.30 & 2039.7 & 8 & 7.8 & م 120 & 5 & 2 & 0 & 8 & 16.0 & & & -23.4 \\
\hline $071858.2+705920$ & 071857.72 & 705921.1 & 10 & 2.6 & 0.082 & 0.22 & 0.27 & 0 & 15.6 & 17.4 & & 0.066 & -20.6 \\
\hline $071923.7+570840$ & 71924.28 & 0839.8 & 0 & 7 & 4 & & 8 & 9 & 4 & 7 & & & -24.1 \\
\hline $072220.6+732041$ & 072222.63 & 732035.3 & 14 & 10.4 & 0.065 & -0.29 & -0.59 & 17 & 16.0 & 17.6 & QSO & 0.350 & -24.1 \\
\hline $072408.4+355309$ & 2408.8 & 55306.2 & 8 & 0 & 0 & 7 & -0.05 & 10 & 1 & 16.6 & & & -23.1 \\
\hline $072826.6+303407$ & 072826.68 & 303412.6 & 12 & 5.7 & 0.069 & 0.55 & 0.27 & 17 & 16.0 & 17.4 & Sey & 0.100 & -21.5 \\
\hline $072912.9+410552$ & 072912.2 & 410550.9 & 10 & 1.0 & 0 & 0.82 & -0.03 & 14 & 15.9 & 17.1 & Ney & .103 & -21.9 \\
\hline \multirow[t]{2}{*}{$073152.4+280423$} & 073152.28 & 280421.2 & 9 & 2.4 & 0.215 & 0.67 & 0.05 & 17 & 15.7 & 17.0 & & & \\
\hline & 073152.72 & 80433.0 & 9 & 10.9 & 0.215 & 0.67 & 0.05 & 17 & 16.5 & 19.1 & 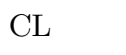 & 0.20 & \\
\hline $073221.5+313750$ & 073221.31 & 313741.4 & 11 & 8.9 & 0.364 & 0.81 & 0.34 & 55 & 16.6 & 20.7 & CL & 0.17 & \\
\hline $073308.7+455511$ & 073309.27 & 455505.6 & 0 & 8.0 & 0.273 & 0.00 & 0.14 & 13 & 15.5 & 16.2 & QSO & 0.142 & -23.5 \\
\hline $073720.4+351752$ & 73720.94 & 351740.9 & 14 & 2.9 & 0.090 & 0.64 & 0.57 & 0 & 15.8 & 18.9 & BL can & 0.213 & \\
\hline
\end{tabular}


Table 2. continued

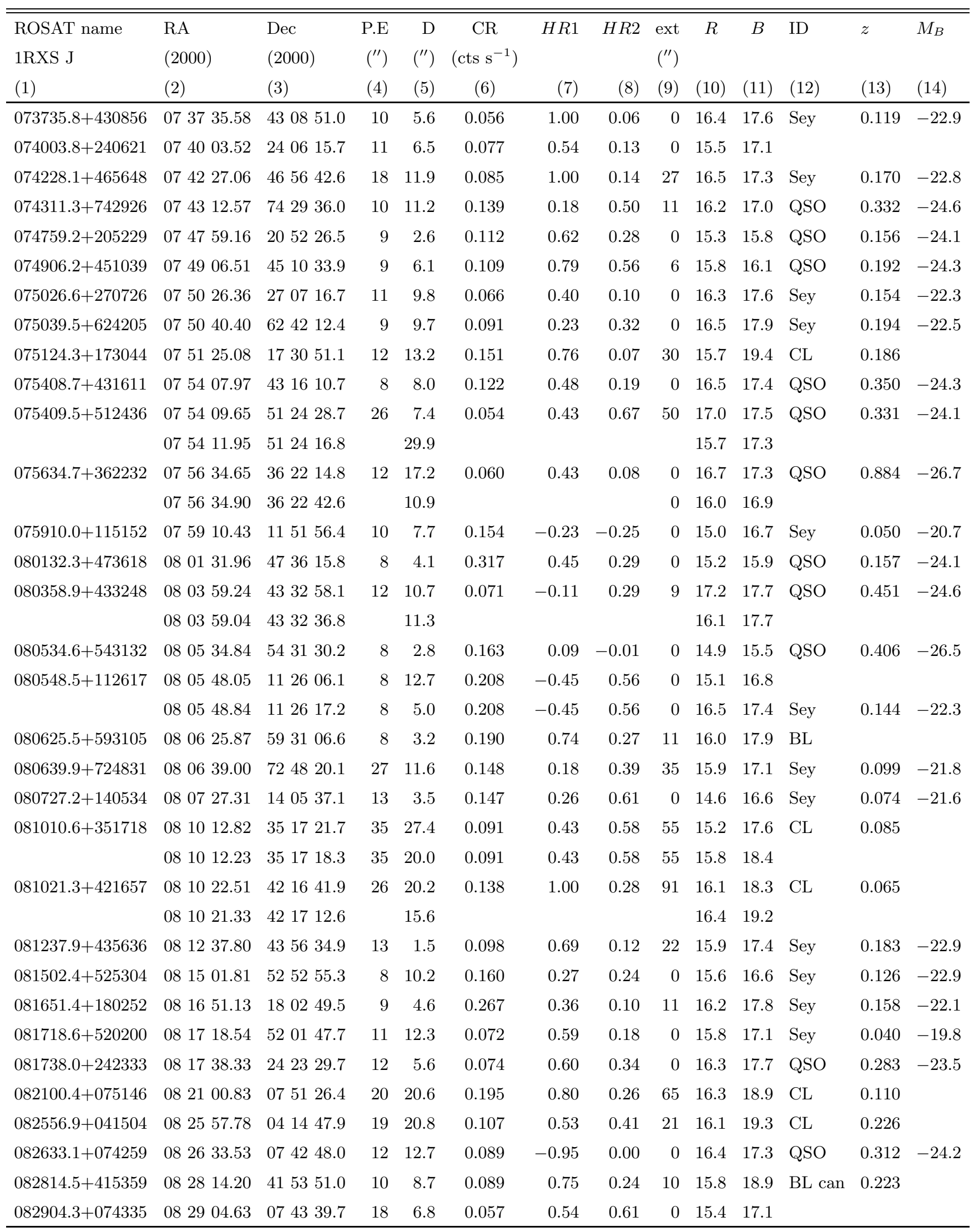


Table 2. continued

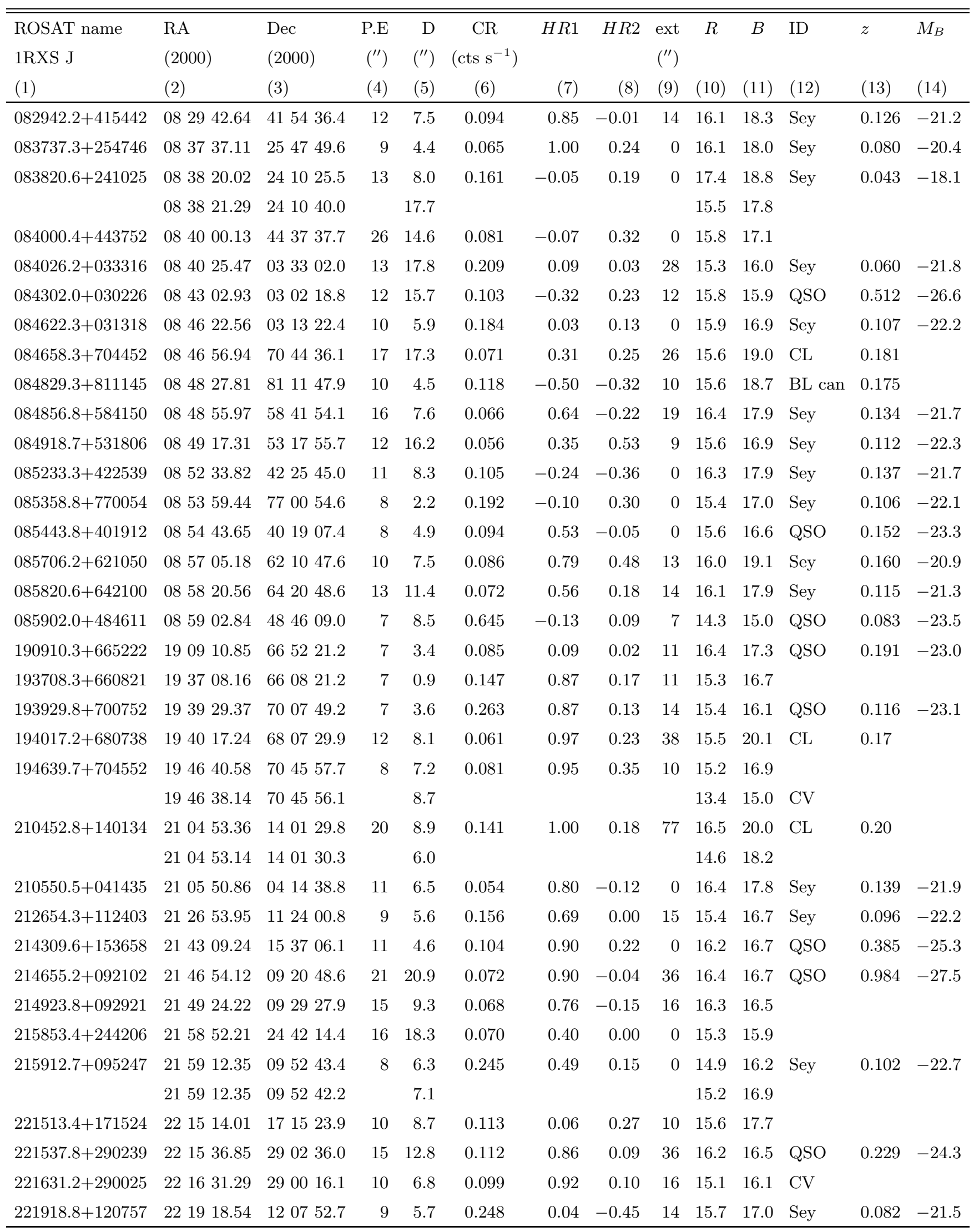


Table 2. continued

\begin{tabular}{|c|c|c|c|c|c|c|c|c|c|c|c|c|c|}
\hline $\begin{array}{l}\text { ROSAT name } \\
\text { 1RXS J } \\
(1)\end{array}$ & $\begin{array}{l}\text { RA } \\
(2000) \\
(2) \\
\end{array}$ & $\begin{array}{l}\text { Dec } \\
(2000) \\
(3) \\
\end{array}$ & $\begin{array}{l}\text { P.E } \\
\left({ }^{\prime \prime}\right) \\
(4)\end{array}$ & $\begin{array}{r}\mathrm{D} \\
\left({ }^{\prime \prime}\right) \\
(5)\end{array}$ & $\begin{array}{c}\mathrm{CR} \\
\left.\operatorname{cts~s}^{-1}\right) \\
(6)\end{array}$ & $H R 1$ & $H R 2$ & $\begin{array}{l}\text { ext } \\
\left({ }^{\prime \prime}\right) \\
(9)\end{array}$ & $(10)$ & $(11)$ & $(12)$ & 13) & $(14)$ \\
\hline 2219 & 21945 & 212048.7 & 10 & 10.5 & 0.080 & 0.34 & 0.21 & 0 & 15.6 & 17.0 & BL can & 0.20 & \\
\hline 2225 & 225 & 6.3 & 12 & 9.3 & .060 & 0.39 & 0.23 & 9 & 16.1 & 16.1 & 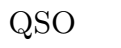 & 173 & -24.1 \\
\hline \multirow[t]{2}{*}{$222657.0+254946$} & 222655.92 & 255009.1 & 25 & 27.3 & 0.101 & 1.00 & -0.12 & 82 & 15.7 & 19.7 & CL & 0.17 & \\
\hline & 22265 & 254939.1 & & 14.4 & & & & & 17.3 & 18.0 & & & \\
\hline $222846.3+$ & 222846.34 & 333507.8 & 8 & 7.8 & 0.113 & 0.66 & 0.33 & 5 & 14.9 & 15.9 & Sey & .090 & -22.8 \\
\hline $222934.3+305720$ & 222934.15 & 305712.1 & 12 & 8.1 & 0.073 & 0.79 & -0.02 & 19 & 16.4 & 17.1 & QSC & 319 & -24.4 \\
\hline $223019.5+$ & 223019.67 & 163112.4 & 8 & 3.0 & 0.159 & 0.54 & 0.26 & 8 & 15.0 & 16.0 & Sey & 084 & -22.5 \\
\hline $224621.7+314206$ & 224621.68 & 314207.6 & 8 & 1.6 & 0.146 & 0.83 & -0.07 & 4 & 15.8 & 17.1 & Sey & 145 & -22.6 \\
\hline $224939.6+$ & 224939.57 & 110029.2 & 9 & 13.2 & 0.184 & 0.44 & 0.07 & 0 & 14.8 & 15.7 & Sey & 884 & -22.8 \\
\hline $225148.5+341937$ & 225147.79 & 341930.3 & 7 & 11.0 & 0.254 & 0.83 & 0.04 & 10 & 16.3 & 16.8 & Sey & 132 & -22.8 \\
\hline 225209.6 & 225209.37 & 264240.7 & 9 & 5.6 & 0.133 & 0.45 & -0.04 & 0 & 15.2 & 16.0 & Sey & 69 & -22.1 \\
\hline \multirow[t]{2}{*}{$225453.7+241449$} & 225453.54 & 241441.0 & 14 & 8.3 & 0.064 & -0.27 & 0.16 & 0 & 16.1 & 17.8 & & & \\
\hline & 225455.03 & 241445.3 & & 18.6 & & & & & 13.5 & 16.3 & M & & \\
\hline 225639.4 & 225639.18 & 261843.1 & 9 & 4.1 & 0.055 & 0.77 & 0.22 & 0 & 16.2 & 18.1 & Sey & 176 & -22.1 \\
\hline $230325.2+075642$ & 230 & 075643.6 & 15 & 2.9 & 0.165 & 0.68 & 0.32 & 41 & 16.2 & 18.9 & CL & 0.16 & \\
\hline $230328.0+144341$ & 230327.92 & 144349.0 & 15 & 8.1 & 0.109 & 0.96 & 0.16 & 11 & 14.7 & 16.4 & Sey & 0.080 & -22.0 \\
\hline $230949.6+213523$ & 230949.28 & 213519.0 & 9 & 6.0 & 0.212 & 0.20 & 0.30 & 18 & 15.7 & 17.9 & $\mathrm{CV}$ & & \\
\hline $231107.7+113812$ & 231106.14 & 113829.2 & 29 & 28.4 & 0.065 & 1.00 & 0.61 & 62 & 16.5 & 20.2 & CL & 0.26 & \\
\hline $231456.0+224325$ & 231455.89 & 224323.4 & 9 & 2.2 & 0.109 & 0.82 & 0 & 10 & 15.7 & 16.5 & $\Omega$ & 1 & -23.6 \\
\hline $231517.5+182825$ & 231517.08 & 182814.4 & 11 & 12.2 & 0.085 & 0.72 & 0.64 & 0 & 15.0 & 16.5 & Sey & 0.104 & -22.5 \\
\hline $232339.1+090842$ & Q 22 י2 22 & - & 23 & 8.8 & 128 & 047 & 0.24 & 71 & 14.8 & 19.9 & CL & 0.21 & \\
\hline $232554.6+215310$ & 232554.24 & 215314.2 & 7 & 6.5 & 0.555 & 0.63 & 0.15 & 5 & 15.5 & 15.8 & QSO & 0.120 & -23.5 \\
\hline 232841. & 202041.41 & 24 T6 & 19 & 5.1 & 0.082 & 0.72 & -0.01 & 24 & 16.4 & 18.0 & Ney & 0.129 & -21.5 \\
\hline $232953.9+062814$ & 232954.38 & 062810.2 & 11 & 8.1 & 0.064 & 0.46 & 0.84 & 0 & 15.6 & 16.6 & $\mathrm{CV}$ & & \\
\hline 2331 & 233151.98 & 195648.0 & 43 & 47.8 & 0.993 & 0.05 & 0.11 & 0 & 13.6 & 15.1 & $\mathrm{~N}$ & & \\
\hline $233315.8+225539$ & 233315.61 & 225549.5 & 26 & 10.8 & 0 & 0.96 & .17 & 86 & 16.4 & 18.6 & CL & 0.07 & \\
\hline 23 & 3 & 0 & 13 & 17.0 & $r$ & 0.96 & 0 & 106 & 13.5 & 15.9 & CL & 0 & \\
\hline $234728.8+242743$ & 234728.77 & 242745.8 & 7 & 2.8 & 0.262 & 0.75 & 0.26 & 10 & 15.9 & 16.5 & QSO & 0.159 & -23.5 \\
\hline $234734.6+271910$ & 234735.24 & 271900.6 & 13 & 12.7 & 0.071 & 0.93 & 0.45 & 0 & 15.9 & 16.1 & QSO & 0.646 & -27.0 \\
\hline $235034.7+292924$ & 235035.65 & 292919.7 & 12 & 13.0 & 0.122 & 0.87 & -0.04 & 0 & 16.0 & 19.5 & CL & 0.153 & \\
\hline $235152.7+261938$ & 235152.87 & 261932.1 & 8 & 6.3 & 0.338 & 0.50 & 0.17 & 9 & 14.9 & 16.6 & sey & 0.040 & -21.3 \\
\hline $235754.3+132418$ & 35753.82 & 132409.6 & 9 & 11.0 & 0.235 & 0.13 & 0.03 & 14 & 15.3 & 15.8 & QSO & 0.115 & -23.4 \\
\hline
\end{tabular}

The abbreviations have the following meanings:

"QSO" : quasi-steller object (unresolved or resolved object with $M_{B}<23$ showing a Seyfert 1 spectrum).

"Sey": Seyfert galaxy.

"BL": BL Lac object.

"BL can": BL Lac candidate.

"CL": cluster of galaxies.

"WD": white dwarf.
"CV": cataclysmic variable star.

"M": M star.

Column (13): Average redshift for extragalactic counterpart.

Column (14): Absolute $B$ magnitude calculated with the formula used by Véron-Cetty \& Véron (1996) for emission line AGN. 
Acknowledgements. We would like to thank L. Cao and X.J. Jiang for their helpful support during the observations. We also thank the anonymous referee for valuable comments and suggestions. The ROSAT project is supported by the Ministerium für Bildung, Wissenschaft, Forschung und Technologie (BMBF/DARA) and by the Max-Planck-Gesellschaft (MPG). This research made use of the NASA/IPAC Extragalactic Database (NED), which is operated by the Jet Propulsion Laboratory, California Institue of Technology, under contract with the National Aeronautics and Space Administration.

\section{References}

Appenzeller I., Thiering I., Zickgraf F.-J., et al., 1998, ApJS 117, 319

Bade N., Fink H.H., Engels D., 1994, A\&A 286, 381

Condon J.J., Cotton W.D., Greisen E.W., et al., 1998, AJ 115, 1693

Fischer J.-U., Hasinger G., Schwope A.D., et al., 1998, Astron. Nachr. 319 (6), 347

Ghisellini G., Maraschi L., Tanzi E.G., Treves A., 1986, ApJ 310,317

Hasinger G., Fischer J.-U., Schwope A.D., et al., 1998, Astron. Nachr. 318 (6), 329

Irwin M., Maddox S., McMahon R., 1994, Spectrum 2, 14
Maccacaro T., Gioia I., Wolter A., et al., 1988, ApJ 326, 680

Massey P., Strobel K., 1988, ApJ 328, 315

Monet D.G., et al., 1996, USNO-A1.0 (Washington DC, USNO)

Nass P., Bade N., Kollgard R.I., et al., 1996, A\&A 309, 419

Pietsch W., Bischoff K., Boller Th., et al., 1998, A\&A 333, 48

Schimdt M., Hasinger G., Gunn J., et al., 1998, A\&A 329, 495

Stocke J., Liebert J., Schmidt G., Gioia I., Maccacaro T., Schild R., Maccagni D., Arp H., 1985, ApJ 298, 619

Stocke J.T., Morris S.L., Gioia I.M., et al., 1991, ApJS 76, 813

Tananbaum H., et al., 1979, ApJ 234, L9

Thomas H.-C., Beuermann K., Reinsch K., et al., 1998, A\&A 335,467

Véron-Cetty M.-P., Véron P., 1996, ESO Scientific Report, No. 17

Voges W., 1992, in Proceedings of the ISY Conference "Space Sciences", ESA ISY-3, ESA Publications, p. 9

Voges W., Aschenbach B., Boller Th., et al., 1996a, IAU Circ. 6420

Voges W., Boller Th., Dennerl K., et al., 1996b, MPE-Report 263, 637

Voges W., 1997, The All-Sky Survey and Pointing Catalogues of ROSAT. In: Di Gesu V́., Duff M.J.B., Heck A., et al. (eds.) data Analysis in Astronomy V. World Sci. Publ., Singapore, p. 189

Yan H.J., 1995 (private communication) 